UCRL-TD- 119298

\title{
Characterization of Mixed Waste For \\ Sorting And Inspection Using Non-Intrusive Methods
}

\author{
G. Patrick Roberson, Richard W. Ryon \\ and Nathan L. BuT1
}

\section{December 1994}

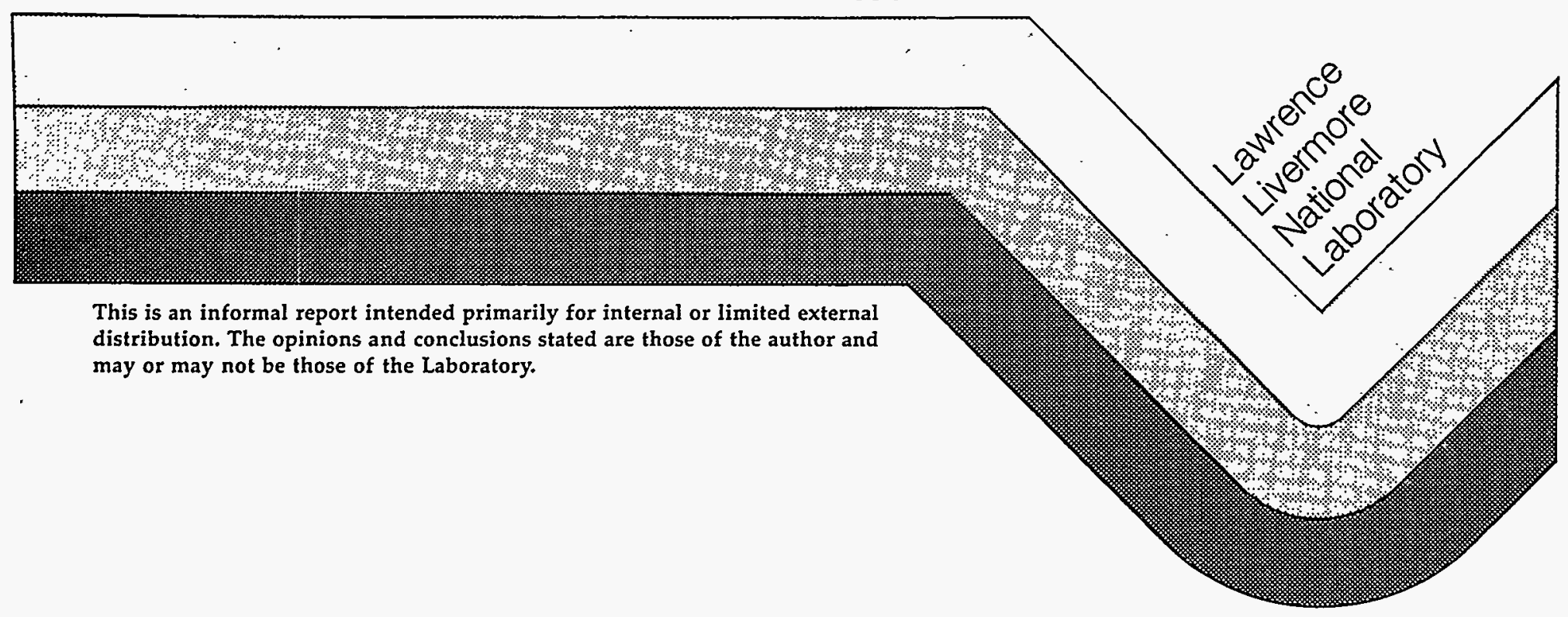




\section{DISCLAIMER}

This document was prepared as an acccount of work sponsored by an agency of the United States Government. Neither the United States Government nor the University of California nor any of their employees, makes any warranty, express or implied, or assumes any legal liability or responsibility for the accuracy, completeness, or usefulness of any information, apparatus, product, or process disclosed, or represents that its use would not infringe privately own rights. Reference herein to any specific commercial products, process, or service by trade name, trademark, manufacturer, or otherwise, does not necessarily constitute or imply its endorsement, recommendation, or favoring by the United States Government or the University of California. The views and opinions of authors expressed herein do not necessarily state or reflect those of the United States Government or the University of California, and shall not be used for advertising or product endorsement purposes.

This report has been reproduced directly from the best available copy.

Available to DOE and DOE contractors from the Office of Scientific and Technical Information P.O. Box 62, Oak Ridge, TN 37831

Prices available from (615) 576-8401, FTS 626-8401

Available to the public from the

National Technical Information Service

U.S. Department of Commerce

5285 Port Royal Rd.

Springfield, VA 22161 


\section{DISCLAIMER}

Portions of this document may be illegible in electronic image products. Images are produced from the best available original document. 


\title{
ChaRACTERIZATION OF MIXED WASTE FOR SORTING AND INSPECTION USING NON-INTRUSIVE METHODS
}

\author{
G. Patrick Roberson, Richard W. Ryon, and Nathan L. Bull \\ Lawrence Livermore National Laboratory \\ Livermore, CA 94551, USA
}

\begin{abstract}
Characterization of mixed wastes (that is, radioactive and otherwise hazardous) requires that all hazardous, non-conforming, and radioactive materials be identified, localized, and quantified. With such information, decisions can be made regarding whether the item is treatable or has been adequately treated. Much of the required information can be gained without taking representative samples and analyzing them in a chemistry laboratory. Non-intrusive methods can be used to provide this information on-line at the waste treatment facility. Ideally, the characterization would be done robotically, and either automatically or semi-automatically in order to improve efficiency and safety.

For the FY94 Mixed Waste Operations (MWO) project, a treatable waste item is defined as a homogeneous metal object that has external radioactive or heavy metal hazardous contamination. Surface treatment of some kind would therefore be the treatment method to be investigated. We developed sorting and inspection requirements, and assessed viable non-intrusive techniques to meet these requirements. We selected radiography, computed tomography and $\mathrm{x}$-ray fluorescence. We have characterized selected mock waste items, and determined minimum detectable amounts of materials. We have demonstrated the efficiency possible by integrating radiographic with tomographic data. Here, we developed a technique to only use radiographic data where the material is homogeneous (fast), and then switching to tomography in those areas where heterogeneity is detected (slower). We also developed a tomographic technique to quantify the volume of each component of a mixed material. This is useful for such things as determining ash content. Lastly, we have developed a document in MOSAIC, an Internet multi-media browser. This document is used to demonstrate the ability to share data and information world-wide.
\end{abstract}




\section{INTRODUCTION}

Characterization of mixed wastes (which are both radioactive and otherwise hazardous) requires that all hazardous and non-conforming materials and radioactive sources be identified and localized. Comprehensive and accurate characterization can provide the information required to determine if a waste item is treatable or has been adequately treated. Much of the required information can be provided without the need to take representative samples to an analytical laboratory. The final waste form must meet federal EPA, state, and other applicable regulatory requirements and do so in a cost effective manner.

The characterization effort of the Lawrence Livermore National Laboratory (LLNL) FY94 Mixed Waste Operations (MWO) project focused on non-intrusive techniques. In the MWO functional flow diagram shown in Figure 1, characterization is performed on waste objects before sorting and treatment and during the inspection process to verify that treatment was adequate. In Figure 1, the two areas that require characterization are outlined in red. The characterization effort incorporated several key tasks that will be discussed in this document. This year we:

- Developed requirements for sorting and inspection of individual waste items,

- Assessed and selected viable characterization technologies,

- Designed a characterization system to achieve the sorting and inspection requirements,

- Characterized selected MWO mock waste items,

- Determined and verified the minimum detectable limits of the technologies used,

- Explored the integration of measurements from different methods (data fusion) to increase speed and accuracy of the characterization process,

- Developed a document in MOSAIC, an Internet-based global multi-media browser. This document is used to demonstrate the ability to share data and information world-wide.

Additionally, it was necessary to pursue several key research elements that provided the necessary techniques needed to achieve the established sorting and inspection requirements. These research elements included:

- Determining homogeneity using radiographic and computed tomography data

- Identification of material using computed tomography data,

- Determining volume using 3D computed tomography data,

- Determine minimum detection limits when using radiography/tomography and $\mathrm{x}$-ray fluorescence.

For the FY94 MWO project, a treatable waste item is defined as a homogeneous metal object that has external radioactive or heavy metal hazardous contamination. These objects are separated and treated so that the amount of mixed waste being disposed of is reduced and valuable metals can be recycled. It is also desirable to separate, treat, and remove metal objects from the waste stream that produce large amounts of ash content in treatment systems like plasma hearth decomposition or vitrification. From the definition of a treatable object, the requirements for sorting and inspection of individual waste items were determined. From the sorting and inspection requirements, applicable characterization technologies were selected and a characterization system was designed. The characterization technologies incorporated in the system were used to characterize selected mock waste items to separate them according to the sorting and inspection requirements. Ideal minimum detectable limits (MDL's) for two technologies were calculated. Data were collected from the characterization systems and used to verify the MDL calculations. Data were also collected from the characterization system and used to determine object composition and material volume. 


\section{TeChNICAL APPROaCh}

\section{Requirements for sorting and inspection}

In preparation for determining sorting and inspection requirements it is necessary to understand the definition of waste items as defined by the EPA. Individual waste items that were characterized are generic "debris" or "containers" as defined by the EPA. The EPA defines "debris" as solid material exceeding $60 \mathrm{~mm}$ particle size intended for disposal and is:

- A manufactured object, or

- Plant or animal matter, or

- Natural geologic material.

Further, any mixture where the debris portion comprises the largest amount of material present by volume as determined by visual inspection is classified as debris. There are four primary subcategories under the EPA debris definition:

- Metals (metal content greater than 95\%),

- Inorganic non-metals,

- Combustibles,

- Heterogeneous.

The performance requirements for physical and/or chemical extraction of hazardous materials from debris to be "a clean surface" is defined as follows: "A clean debris surface is a surface that, when viewed without magnification, shall be free of all visible contaminated soil and hazardous waste, except that residual staining caused by soil and waste consisting of light shadows, slight streaks, or minor discolorations, and soil and waste in cracks, crevices, and pits may be present provided that such staining and soil and waste in cracks, crevices, and pits shall be limited to no more than 5\% of each square inch of surface area." [BRN93]

The definition of a "container" is any vessel that retains $75 \%$ of it's original volume. Containers are not debris and therefore are not subject to the treatment standards for debris. However, containers that have been damaged or are otherwise not intact and nonfunctional are classified as debris. These containers are subject to residues of hazardous waste in empty containers ( ${ }^{*} 40 \mathrm{CFR} \$ 261.7$ defines what an empty container is). If there is no further use for a container, and it is properly emptied, there is no requirement to further clean the container of hazardous residue. The container can be disposed of in a municipal landfill or low-level radioactive waste disposal facility.

Unfortunately, the definitions for clean debris and empty containers are subjective and not quantitative. If the mixed waste characterization technologies are to be automated, the clean debris and empty container rules must have quantitative bounds. Since there is no definitive bounds, we have determined the approximate minimum detectable limits for several of the technologies used for this project and report them.

\footnotetext{
* 40CFR \$261.7: All wastes have been removed that can be removed using the practices commonly employed to remove materials from that type of container, e.g., pouring, pumping, and aspirating and no more then 2.5 centimeters of residue remain on the bottom of the container or inner liner or no more then 3 percent by weight of the total capacity of the container remains in the container or inner liner if the container is less then or equal to 110 gallons in size.
} 
Only homogeneous metal debris and containers are separated from the mixed waste stream for treatment during this phase of the MWO project. From the definition of a treatable object for this project, non-intrusive characterization methods will be used to classify waste items according to the following sorting and inspection requirements:

- Any non-conforming waste items such as liquids, combustibles, or pressurized containers are removed from the waste treatment stream,

- Any objects that are not metal or objects that have unknown composition are removed from the treatment waste stream,

- Any objects that are not homogeneous are removed from the waste treatment stream,

- Any objects that have internal radioactive or hazardous contamination are removed from the waste treatment stream.

For this portion of the MWO project, only heavy metal (e.g. silver, cadmium, barium, mercury and lead) are considered as hazardous materials. Additionally, the characterization system is designed to evaluate metal type and ash content by determining object composition and material volume. The input to the characterization system is the object to be evaluated and is supplied by either the singulation or treatment functions as shown in Figure 1. The output of the characterization system is either a treatable object supplied to treatment, non-treatable object, object to be further singulated, or a clean object that need not be treated.

\section{Assessment and selection of viable non-intrusive characterization technologies}

We distinguish here between nondestructive evaluation (NDE) and nondestructive assay (NDA). Evaluation includes methods which allow observation of inhomogeneities and identification of materials. Assay implicitly means quantitative analysis. We chose existing NDE and NDA technologies that are available here at LLNL to perform our demonstration. Eddy current, $x$-ray imaging, and gamma-ray-spectroscopy methods were evaluated for non-intrusive characterization of individual mock mixed-waste items to satisfy the sorting and inspection requirements discussed above. The NDE and NDA methods used for characterization of various mock waste items are:

NDE/NDA without using $x$-rays:

- ECMD - Eddy Current Metal Detection (located in B166) (work performed in FY93).

NDE/NDA using x-rays:

- RTR - Real-Time Radiography,

- DR - Digital Radiography,

- TCT - Transmission Computed Tomography,

- XRF - X-Ray Fluorescence.

NDA using gamma-ray spectroscopy:

- SRD - Spectroscopy Radiation Detection,

- A\&PCT - Active and Passive Computed Tomography. below.

Each of the technologies used on the MWO characterization system are briefly described 


\section{Eddy current metal detector}

Eddy current techniques offer low-cost methods for high speed, large scale inspection of metallic materials. Eddy current methods can be used for both quantitative measurements of material properties and sorting of objects by dimensions, properties or presence of discontinuities. The Eddy current metal detector used for characterization in FY93 is shown in Figure 2. [GRA94]

Eddy current methods for nondestructive, non-contact evaluation of electrically conducting materials rely on the principles of magnetic induction. When a coil excited by an alternating current is brought in close proximity to a conducting material, the terminal impedance of the coil changes. This change in impedance is associated with the fact that the primary field set up by the eddy current coil induces eddy currents within the electrically conducting material. The eddy currents induced in the material generate their own magnetic field. The magnitude, time lag, phase angle and flow patterns of eddy currents within the material under test are detected by measuring the resultant magnetic field with another set of sensing coils or solid state magnetic field detectors.

\section{$R T R, D R$ and $T C T$}

Conventional $\mathrm{x}$ - or gamma-ray imaging methods to the first order measure the attenuation of an incident beam that travels in a straight path through an object. For NDE methods using RTR, $\mathrm{DR}$, and TCT, the data measured are photon intensities of the incident beam and of the transmitted beam. This is true to first order, ignoring the effects of scattering. The incident beam is partially absorbed and scattered in the object of interest with the remaining transmitted photons traveling in straight lines to the detector plane where they are recorded. The result is a gray-scale shadow, such as a medical $\mathrm{x}$-ray. The quantity that is recorded at each $\mathrm{x}, \mathrm{y}$ coordinate of the detector plane for RTR and DR imaging is the intensity transmission through the thickness of the object, integrated over the spectrum of the incident radiation. The system shown in Figure 3a can acquire both a digital radiographic and computed tomography images. The images shown in the figure are both digital radiographs of mock waste objects and will be described in detail later.

Radiographs are two-dimensional images of three-dimensional objects. That is, radiographs represent the superposition of all features within the object as shown in Figure 4a. However, transmission computed tomography provides a true three dimensional representation of a plane extracted from the object as shown in Figure 4a. For TCT, the digitized radiographic images of the object are recorded at many different angles; cross-sectional images of the object are reconstructed using a computer. The quantity that is reconstructed is the attenuation value for some volume element, or voxel, at location $x=(x, y, z)$ within the object. The voxel is defined by scan and image reconstruction parameters. The reconstruction algorithms require line integrals, also called ray sums, for many ray paths through the object. It is useful to note that the CT ray sum is analogous to a simple gamma-ray transmission gauge measurement. Figure 4 shows several tomograms acquired from waste objects and will be discussed in more detail later.[MAR94, REI92, \& KAW90]

\section{$X$-Ray Fluorescence (XRF)}

$\mathrm{X}$-ray fluorescence is used to identify chemical elements and their abundances. Except for instances where the material has low $\mathrm{X}$-ray absorption, it is considered a surface analysis technique. XRF can identify the composition of objects if the surface and interior are the same material(s). Likewise, it can identify what is on the surface, whether by intention or inadvertent contamination. The technique can easily identify hazardous heavy metals such as lead, cadmium, and mercury or precious metals such as silver and gold. Unless the surface is exposed and measurements are made in a vacuum, elements below about titanium cannot be detected by their 
fluorescence. A measurement of elastically and inelastically scattered incident radiation can be correlated with the average composition of the material. Such a measurement might identify toxic low atomic number materials such as beryllium if present in sufficient quantity.

A typical x-ray fluorescence system consists of three components: 1.) an excitation source, which can be either $x$-ray tubes or radioisotopes such as ${ }^{109} \mathrm{Cd} ; 2$.) a detector (such as lithium drifted silicon or high purity germanium) and its associated electronics; and 3.) a pulse height analyzer and computer for analyzing the fluorescent spectrum and displaying it if desired. The system can be programmed to automatically flag the presence of materials of interest, such as toxic heavy metals. It is interesting to note that the same germanium detector used to identify radioisotopes may be used as an X-ray fluorescence detector.

X-ray fluorescence was used to characterize waste by identifying the presence of toxic heavy metals. It is also used to assess the state of completion of surface decontamination, either releasing the material for disposal or recycling it for further treatment. Figure 5a is a photograph of an X-ray fluorescence system located in a glove box. The graph in Figure $5 \mathrm{~b}$ shows two spectra taken from the exterior of tin cans One spectrum shows a can which has the interior tin coating intact; both iron and tin fluorescence can be seen. The other can had corroded tin; the spectrum shows that the can has very little tin and mostly iron present.

\section{Spectroscopy radiation detection}

Instead of merely detecting radiation, as with a Geiger counter, spectroscopy radiation detection measures the energy of the gamma rays emitted by any radioactive material. The energy spectrum acts as a "fingerprint" which identifies the individual radioactive isotopes present (for instance ${ }^{241} \mathrm{Am}$ or ${ }^{239} \mathrm{Pu}$ as shown in Figure $6 \mathrm{~b}$ ). Fast library look-up programs are used to make the identifications. The intensity (or number) of each gamma ray is also measured. With a calibrated detector, the intensity is directly related to the absolute amount of the radioisotope present. It is for this reason that spectroscopy radiation detection is the heart of A\&PCT technology described below. Figure 6a shows the typical spectroscopy electronics required for a gamma-ray detection system. Figure $6 \mathrm{~b}$ shows two typical gamma ray spectra acquired from actual radioactive waste. Several lines are identified showing that ${ }^{241} \mathrm{Am}$ and ${ }^{239} \mathrm{Pu}$ isotopes are present. The components necessary to make such a measurement include a high purity germanium detector, its associated amplifiers and pulse processor, a pulse height analyzer, and a computer to display the spectrum, perform the identifications, and for data processing.[DEB88]

\section{Active and Passive Computed Tomography (A\&PCT)}

A\&PCT is an accurate gamma-ray NDA method that can identify all detectable radioisotopes present in a container and provide a quantitative measure of their activity. Figure $7 \mathrm{c}$ is a photograph of an A\&PCT system designed to characterize drums or objects. The active CT (ACT) technology can yield quantitative attenuation data (related to density and atomic number) for the waste matrix within a drum. Passive CT (PCT) can, in principle, localize all detectable radioisotopes within a drum and more accurately determine their identity if an entire energy spectrum is obtained.

For ACT (Figure 7a), the property imaged is the $\mathrm{x}$ - or gamma-ray attenuation of an external source. For the case of PCT (Figure $7 \mathrm{~b}$ ), the property imaged is gamma-ray activity at one or more energies of all detectable radioisotopes within a drum. The gamma rays are attenuated as they pass from the source to the detector. The ACT images are used to correct the PCT images for that attenuation in order to determine the activity of the emitting source. For an A\&PCT scanner with gamma-ray spectrometry detection equipment, each radioisotope in the drum can be identified by the energy of its characteristic peak(s) as described above. Figure 7d shows actual A\&PCT data of 
a waste drum with a radioactive source located inside. The image on the left is the attenuation map of the drum contents and the image on the right is a map of the active source within the drum.[ROB94]

\section{Characterization system design}

Using the requirements for sorting and inspection and the identified NDE/NDA technologies described above, a MWO characterization system was designed for treating homogeneous metal objects. Figure 8 shows a block diagram of the characterization system. The characterization system is designed to be used for both sorting objects before treatment and inspecting objects after treatment. At the top of the figure an object is delivered from the singulation function and enters the characterization system. The first NDE/NDA technologies used for analysis are radiography and transmission computed tomography. All three technologies (RTR, DR and TCT) could be available at a single station. The major components that make up each of the technologies are basically the same. If necessary, RTR, DR and TCT data are acquired. The data are used to determine if nonconforming objects such as liquids, combustibles or pressurized containers are present; if so, they are removed from the treatable waste stream.

The next technology used in the system is eddy current metal detection. When this technology is combined with RTR, DR, and TCT data, ash content and the metal type (if metal) of the waste object can be determined. If the object is not metal, it is determined untreatable and removed from the treatable waste stream. However, if the object is metal then it is evaluated for internal heavy metals and inhomogeneity. If either case is true, the object is returned for further singulation.

After the metal detector, the homogeneous metal object is evaluated for radiation. If the object is found to be radioactive, A\&PCT is performed on the object to determine if the radioactive contamination is internal or external. If the radiation is internal, the object is not treatable and should be evaluated for further singulation. If the radiation is external, the object is treated and re-evaluated for radiation. If radiation is present the second time through the system, the object is retreated without performing A\&PCT. After the object is successfully treated for radioactive contamination or if the object did not have radioactive contamination, it is evaluated for external contamination of hazardous material using $\mathrm{x}$-ray fluorescence. Only heavy metal (e.g. silver, cadmium, barium, mercury and lead) contamination is evaluated. If contamination is present, the object is treated. After treatment the object is re-examined for hazardous contamination and if present the object is retreated. If no contamination is present the object is determined clean and removed from the waste stream. The radiation detection, A\&PCT and x-ray fluorescence technologies could be combined and located at a single station. As with the RTR, DR, and TCT technologies above, these technologies are also made up of the same basic components.

The characterization system was not located in the MWO glove box due to the cost involved in relocating the existing hardware. In a fully functioning mixed waste treatment facility, these functions would be performed on-line and would utilize robotics and automated data processing. Individual mock waste items were manually transferred to the NDE facilities and existing NDE/NDA instruments were used for comprehensive characterization. After the characterization process, the objects that are characterized were manually transferred back to the treatment area. 
All waste items being evaluated are mock, that is they are clean and not contaminated by radioactive or hazardous materials. Typical waste items are determined from analysis of waste drum content codes and included:

- Lead, both brick and sheet,

- Steel pipes,

- Polyethylene bottles,

- Vollrath cans,

- Combustible lab garments and wipes,

- Heterogeneous metals (e.g. electric motor),

- Hand tools,

- Paint cans containing combustibles,

- Ceramic crucible parts.

Radioactive contamination of the waste items are simulated using various sealed check sources (standards). Heavy metal contamination is simulated by taping elemental thin film standards to the objects being evaluated.

\section{EXPERIMENTS, RESULTS, AND DISCUSSION}

\section{Characterization of mock waste items}

Existing NDE systems were used to characterize various individual mock waste items that are representative of DOE waste streams (i.e., metals, combustibles, organics, mixed, and radioactive). Methods were investigated to optimize the characterization of these items and minimize the scan time. The MWO mock waste items were transported to the NDE section for evaluation. Representative results of the characterization work are shown in Figures 3-7. Figure $3 \mathrm{~b}$ shows a radiograph of a typical tool that may be found in the waste stream. This is a radiograph of a mechanics level showing that water is present inside the glass tube of the level. Figure 3c is a ID profile through the image displaying relative intensity verses pixels. Also, Figure $3 \mathrm{~d}$ is a radiograph of a rubber glove, a typical object that may be found in the waste stream.

Figure $4 \mathrm{~b}$ is a collage of TCT images taken of various objects including: booties and screwdrivers, metal frame work, an electric motor, gloves, ceramic pipes and a paint can. The TCT imaging technique provides the information necessary to determine if an object is homogeneous. The electric motor in Figure $4 \mathrm{~b}$ has obvious variations in relative attenuation caused by differences in material composition within the object and is therefore not homogeneous. This aspect of TCT will be discussed in further detail in the next section.

Figure $5 b$ shows an example of data acquired on an $x$-ray fluorescence system that characterizes two juice cans. The two spectra shown in the figure provide information about the object elemental composition and the presence or deficiency of tin. In this example, the object that is being characterized is made of iron and the possible "contaminant" is tin. The blue plot in the figure shows a large amount of tin present on a can, and the red plot shows a can with the tin partially removed.

Figure $6 \mathrm{~b}$ shows spectra acquired on a spectroscopy radiation detection system. This spectra show the presence of radioactive materials and identifies the presence of ${ }^{241} \mathrm{Am}$ and ${ }^{239} \mathrm{Pu}$ from their peaks. Figure 7d shows two images acquired from an A\&PCT scanner. The image on the left is an attenuation map of the materials within the object. The image on the right is a map of the radioactive source within the object. The object under characterization is a waste drum and the source is obviously located inside the drum. This object is determined as untreatable and must be 
further singulated before it is treated. Additionally, the attenuation map is used to correct the passive CT image and an accurate determination of the intensity of radioactive source within the drum is made. Combining the data acquired on the spectroscopy and the A\&PCT systems, the radioactive source is identified, the shape and location of the source is determined and the activity is computed.

\section{Material Identification and Volume Determination}

Radiography produces 2-D images of 3-D objects. Dimensional measurements are difficult if not impossible to determine using radiographic images. However, CT data produces images with true three dimensional information. A 3D reconstructed CT image of an object contains pixels with values representing attenuation. Each pixel also represents a volume element or voxel with known dimensional measurements for a location $(x, y$, and $z$ ) within the object being scanned. Using the TCT attenuation information, materials can be identified by comparing the values to standard references that are either scanned with the object or scanned prior, and the information is accessed from a lookup table. Knowing the number of voxels that make up a material and the dimensions of each voxel, the volume of the material can also be determined.

It is possible to identify materials using 3-dimensional transmission computed tomography data sets. 3D attenuation maps of several representative waste items were reconstructed to determined whether the items contain homogeneous or heterogeneous metals, combustible, or unknown material(s). Figure 9a shows a transmission computed tomography reconstructed image that reveals information about the types and quantities of materials within a DC motor system. The colorbar below the image provides a scale relating the different colors in the TCT image to the attenuation coefficient in $\mathrm{cm}^{-1}$. The attenuation variations are related to changes in density ( $\rho$ ) and/or effective-atomic number ( $Z_{\text {eff }}$ ). Figure $9 b$ shows a $1 D$ profile (black line) through the TCT image revealing variations of the linear attenuation coefficient within the motor. The variations in the $2 \mathrm{D}$ profile clearly indicates that the mock waste object in not homogeneous. The measured attenuation coefficient is compared to theoretically calculated values of various materials (labeled and shown by colored flat lines in Figure $9 \mathrm{~b}$ ). This procedure enables us to determine whether the imaged item contains homogeneous or heterogeneous metals, reasonable estimates of the types of metals present, and whether combustible or unknown material(s) are present.

Additionally, a histogram of the CT image can also be used to identify the materials within the individual waste item and indicate their relative amounts as shown in Figure $9 c$. If the histogram contained only two peaks, one for background (air) and one for steel, the item would be classified as a homogeneous metal. However, the histogram of this CT image contains many peaks and indicates the presence of heterogeneous metals. This information is used to nondestructively determine the type and amount of materials within individual objects and nuclear waste containers. For example, 3D TCT can be used to determine the amount of hazardous materials, liquids, and ash content within objects or waste containers. Below we describe a method for estimating volume using CT data and presents an example using three objects of different materials with well known dimensions.

\section{Experiment}

Three objects of known volume were scanned on a TCT system described below. The three objects consisted of wood, plastic and aluminum. The dimensions for each object are shown in Figure 10b. A rendered image of the 3D TCT data set of the three objects is shown in Figure 10a. The calculated volume of each object is listed in column 2 of the table shown in Figure 11b. The objects were placed on what we call the "LCAT" system so that their entire volume could be scanned. 
LCAT is a CT scanner based on a linear-array that is configured with a current-integrating detector and no collimation (or septa) between adjacent detector elements. LCAT is a rotate-only CT scanner that employs a Thomson THX2023 solid-state linear X-ray detector. The array contains 1024 total detector elements, $0.45 \times 0.6 \mathrm{~mm}$ with a pitch of $0.45 \mathrm{~mm}$ and has a total length of 460 $\mathrm{mm}$ (resulting in no dead space). The detector system is an assembly of 16 modular arrays of 64 photodiodes coated with a scintillator made from gadolinium oxide doped with terbium. The modules are placed side by side where the first photodiode is 30 percent of the area of the other photodiodes to maintain a $0.45 \mathrm{~mm}$ pitch at the joint. The dynamic range of this detector system is $4000 / 1$. The integration time for one CT projection is adjustable from 6 to 100 milliseconds and depends on the available $x$-ray flux and the scanning speed of the stage. Many airport baggage scanners use this type of detector system.

A Phillips $160 \mathrm{kVp}$ x-ray tube with a $0.4 \mathrm{~mm}$ focal spot is used as a source for the LCAT scanner. The maximum source to detector distance is $124.5 \mathrm{~cm}$. The staging consists of a vertical $(\mathrm{z})$ and a rotational $(\theta)$ drive that can perform both digital radiography and CT. Both stages are mounted on a manually operated linear rail that provides geometric enlargement when moving the object closer to the source. Objects to be inspected on the LCAT scanner are rotated in a fan-beam of radiation at a constant velocity. The linear-array detector rapidly samples the radiation beam as the object rotates, acquiring a projection at each sampling location.

The following parameters were used for the volume CT scan of the three test objects. The $\mathrm{x}-$ ray tube was operated at $160 \mathrm{kVp}$ and $1.9 \mathrm{~mA}$ with a $0.4 \mathrm{~mm}$ copper filter. The objects were oversampled in the $\mathrm{z}$-axis by $\sim 73 \%$ to yield a slice thickness of approximately $0.437 \mathrm{~mm}$. The geometric enlargement (for fan-beam $\mathrm{x}$ and $\mathrm{y}$-axis only) was approximately 1.15 , yielding a final image voxel (CT reconstructed images include slice thickness) size of approximately $0.392 \mathrm{~mm} \mathrm{x}$ $0.392 \mathrm{~mm}$ and $0.437 \mathrm{~mm}$ thick. There were $122 \mathrm{CT}$ slices taken along the three objects, each slice consisted of 600 projections over 360 degrees with 360 ray sums in each projection. The integration time for each projection was 100 milliseconds. The data were reconstructed into $122 \mathrm{CT}$ images each containing 189 X 205 pixels using a convolution backprojection algorithm. The reconstructed images were trimmed (the data set was reduced by removing the unnecessary data representing air volume around the three objects) and converted to 8 bit integer (byte) data for 3D rendering. The trimmed reconstructed image used for rendering was $4.7 \mathrm{Mbytes}$ of data.

\section{Results and Discussion}

Two methods of volume determination were used on the 3D data acquired for this experiment. One method uses the histogram of the image to calculated the volume and the other method used segmentation. The results of both methods used in this experiment are shown in Figures 10 \& 11. A representative CT reconstruction of the three object data set is shown in Figure 10c. This reconstruction is a single slice (slice number 47 ) extracted from the CT volume data set. The three objects are labeled in the figure. The different colors in the CT image represent relative attenuation. The attenuation variations are related to changes in density $(\rho)$ and/or effective-atomic number $\left(Z_{\text {eff }}\right)$. Dark orange represents low attenuating objects and bright yellow represents higher attenuating objects.

A histogram of the individual reconstructed tomograms is shown in Figure 10d. The histogram reveals the distribution of relative attenuation values within the three objects and the amount by volume within each slice, since each pixel represents the average attenuation for a volume of $0.392 \mathrm{~mm}^{2}$ and $0.437 \mathrm{~mm}$ thick. Analysis of these data reveals the different attenuation distributions that are related to the different materials that make up the three objects. The peak 
located at highest relative attenuation represents all the pixels within the CT slice that are related to aluminum.

\section{Histogram method}

In the histogram method of determining volume, integrating the area under the peak provides the number of pixels associated with each material. Multiplying this number by the pixel volume size provides the determination of volume for each material within each slice of the 3D CT data set. Performing this calculation for each slice in the 3D data set and summing up the results provides an estimate of the total volume of each material. Using the histogram method is somewhat subjective because the endpoints for each peak are ill-defined.

The calculation described above was performed for each peak shown in the histogram, providing a volume estimate for each of the three materials scanned in this experiment. The table in Figure 11 shows a summery of the results. The first column lists the material that was scanned. The second column lists the ideal volume that was calculated from the dimensions of the object. The third column is the total number of pixels measured for each of the objects scanned using the histogram method. The fourth column is the volume measured for each object scanned and was determined using the histogram pixel summation. The fifth column lists the percent error of measured verses calculated volume for each object using the histogram method.

The histogram results show that the measured volume of the higher attenuating object (aluminum) was underestimated by approximately $16.3 \%$. The measured volume of the plastic object was underestimated by approximately $10.7 \%$ and the measured volume of the wood object was overestimated by approximately $24 \%$. The underestimation is most likely due to the partial volume effect within the CT image. The partial volume effect is observed when a CT detector only measures part of the volume of an object, usually at the edge of an object. The relative attenuation value measured at the edge of an object will vary in magnitude depending on the sampling position of the detector. This effect can be seen in the CT image in Figure 10c. The orange edge around the yellow aluminum square represents the partial volume measurements. The partial volume measurement is lower in magnitude than the relative attenuation of the aluminum. The lower attenuation values caused by the partial volume effect will scatter down the histogram and add to the pixel count of the lower attenuating objects. The partial volume effect causes an underestimation in the volume determined for higher attenuating objects and an over-estimation in the volume determination of lower attenuating objects.

A higher number of objects with greater surface area will produce larger amounts of error due to the partial volume effect. The partial volume effect will have a minimum effect on volume measurements if the objects within the volume data set are segmented and separated from each other. For a more accurate determination of volume, it is necessary to segment and separate the objects before the volume determination is made.

\section{Segmentation method}

Segmentation is used to group pixels into regions of objects based on intensity similarity and connectivity. Connectivity is a method that creates partitions in an image based on a pixel's neighborhood. By segmenting the objects in a 3D image, intensity overlap from other objects in the image is reduced. In order to automate the segmentation process, noise has to be reduced in the 
image. Analysis takes place after each object is isolated. The basic algorithm that was developed for determining volume from a $3 \mathrm{D}$ data set is as follows:

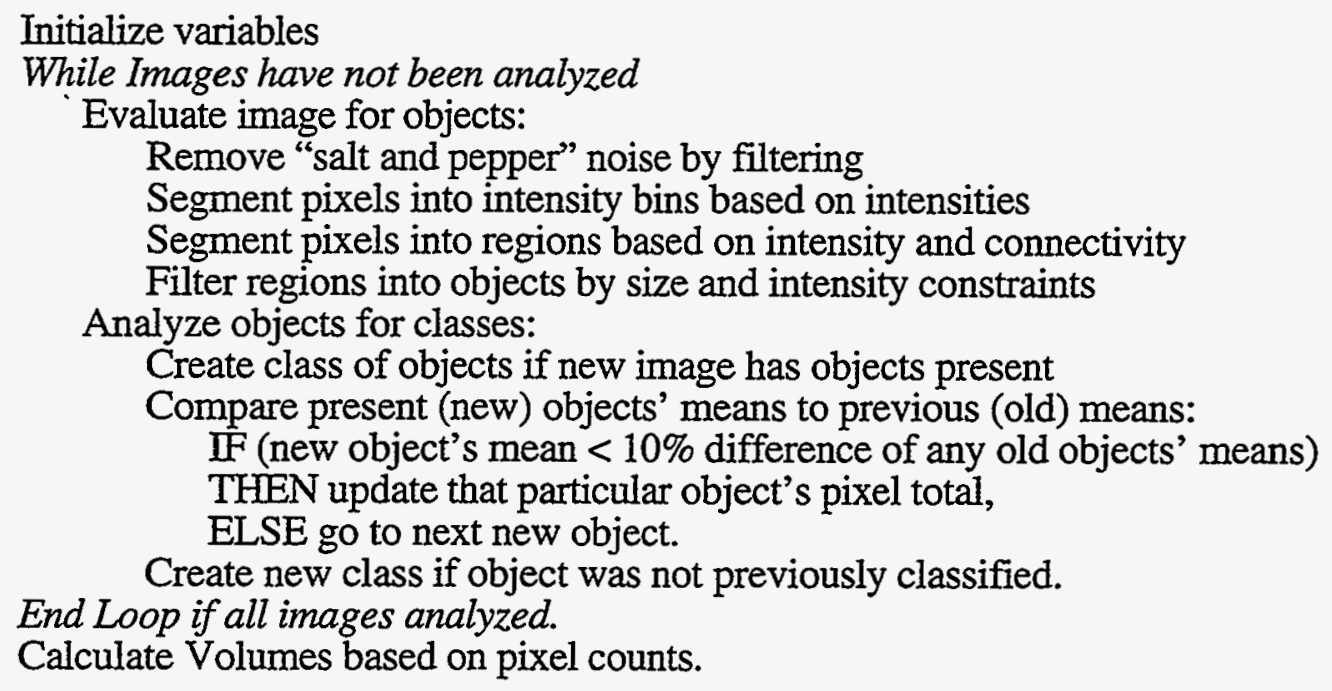

Note that each step in the algorithm is performed on every TCT image individually. The advantages of analyzing each TCT slice individually instead of the entire 3D data set are: 1.) analyzing a single slice from the volume set is far less computationally expensive than analyzing the entire volume data set, and 2.) the amount of memory needed to store the several 3D data sets needed during analysis is far greater than a that needed for a single slice.

The procedure described above was implemented on the mock-waste items and the results are shown in Figure 11. Figure 11a shows a collection of the three objects that were segmented and extracted from the $3 \mathrm{D}$ volume. The results are listed in the table of Figure $11 \mathrm{~b}$. The sixth column of the table lists the total pixel count found for each object using the segmentation method. The seventh column lists the estimated volume calculated from the results of column six and the amount of volume each pixel represents. The eighth column is the percent error between the calculated ideal and TCT measured volume for each object.

The segmentation procedure provides very good results when determining the volume of objects using 3D TCT data sets. All of the measured volumes are close to, but slightly less than the ideal values of the objects' dimensions. The greatest source of error is most likely from the missclassification of the objects' edge pixels caused by the partial volume effect. This missclassification reduced the objects' pixel totals which lead to a reduction in the objects' estimated volumes.

\section{Technology Integration}

NDE/NDA technology integration provides three major benefits in the characterization system. First, integration of two or more technologies can provide information that is more accurate than any one technology can provide on its own. Second, there may be information that can be extracted from the combination of two or more technologies that does not exist in any single technology. Third, technology integration can greatly speed up the acquisition process. Here we give two examples of technology integration, active and passive tomography and digital radiography coupled with transmission computed tomography. 


\section{A\&PCT Integration}

A\&PCT is an example of the integration of three technologies that provide information that is not otherwise available. Using active tomography, the attenuation of the contents of an object are mapped. The image on the left of Figure $7 \mathrm{~d}$ is an attenuation map of a waste drum. Using passive tomography, the activity of any radioactive source is mapped. The image on the right in Figure 7d is an emission map of a point source located in the same drum. Knowing the location of the source and attenuators around the source provides information that is necessary to compensate the source for attenuation and make an accurate determination of the source strength. Spectroscopy radiation detection provides the identification of the radioisotope within the object or drum. The combination of the three technologies provide information that reveals the radioactive sources location, shape, identity, and intensity.

\section{$D R \& T C T$ Integration}

The combination of data from digital radiography and computed tomography can be used to speed up the process of determining if an object is homogeneous. Figure 12a shows an object that was scanned using radiography and TCT. The object is a simulated glove box section that is made up of two plates of Lexan and one plate of aluminum. Figure 12b is a radiograph of the simulated glove box section. From the radiograph it is difficult if not impossible to tell the difference between attenuation caused by object geometry (thickness) or material composition. Transmission computed tomography can reveal this distinction. However, a full 3D scan of the object is time consuming. It is desirable to perform a minimum amount TCT on the object to reduce the acquisition and analysis time required to determine homogeneity.

In Figure $12 \mathrm{c}$ the radiographic image is shifted along the $y$ axis and subtracted from itself. The image is summed along the $\mathrm{x}$ axis and the result is shown in Figure 12d. Each peaks in the figure shows where there is a change in the attenuation value of the image along the $y$ axis. The larger the peak the greater the difference in attenuation from one region to the next. This information can be used to determine how many TCT images are necessary to determine homogeneity and where the scans should be located. Figure 12e shows three TCT scans acquired in three different regions where the attenuation changes in the radiograph. The TCT scans show that one region of attenuation change is due to a change in the geometry of the object and the other attenuation difference is due to a change in object material. This object is determined to be inhomogeneous as determined by only three TCT scans. The variation of the 1D profile in Figure $12 \mathrm{f}$ verifies that there is a change in the simulated glove box material.

\section{Detection Limits using X-Ray Radiography and Fluorescence}

In both waste characterization prior to treatment and in inspecting objects after treatment, it is necessary to have reasonable estimates of the amounts of hazardous materials which can be found. The answer is complex, because it depends upon both the material to be detected and the material on which or within which it is located. The answer also depends upon the size of the object being inspected if the sought material is not on the surface. We confine the discussion here to inorganic substances, and in particular to heavy metals, as the detection of hazardous organic materials requires sampling and chemical analysis. We look here at two radiation technologies for materials characterization, namely digital radiography and $\mathrm{x}$-ray fluorescence. 


\section{Radiography:}

Radiography is particularly useful as a screening tool. The technique can quickly determine possible inhomogeneities which may represent hazardous materials. Such suspect regions in a whole barrel or within a segregated object can then be investigated further, for instance by computed tomography, $\mathrm{x}$-ray fluorescence, or chemical analysis. The question is, how much of the hazardous substance can be detected?

Our approach to answer this question is to look at the range of materials which typically are found in waste streams. We have used plastic (to represent general trash, such as paper, garments, and other low attenuating materials), aluminum (to represent such things as construction materials like concrete, wallboard, and other medium attenuating materials) and steel (to represent heavy materials from construction and machinery which attenuate $x$-rays strongly).

The mathematics are pretty simple. Ideally, radiation attenuation is given by

$$
\left(\mathrm{I} / \mathrm{Io}_{1}\right)_{1}=\exp \left(-\mu_{1} \rho_{1} \mathrm{x}_{1}\right)
$$

where $I / I_{0}$ is the measured attenuation of the incident radiation of intensity Io, $\mu$ is the mass attenuation coefficient for the material at a specific energy of radiation, $r$ is the density, and $x$ is the thickness. The subscript 1 is used here to designate the primary, or matrix, material. If a second (hazardous or toxic) material is added, the attenuation of x-rays is changed to be

$$
(\mathrm{I} / \mathrm{To})_{2}=\exp \left(-\mu_{1} \rho_{1} \mathrm{x}_{1}\right) \cdot \exp \left(-\mu_{2} \rho_{2} \mathrm{x}_{2}\right)=(1 \pm f) \times(\mathrm{I} / \mathrm{Io})_{1}
$$

where the subscript 2 refers to the added material, and the letter $f$ refers to the fractional change in contrast which can be detected (in the range of <.01 to >.03). Solving equations 1 and 2 for the thickness of added material, we find that

$$
x_{2}=-\ln (1 \pm f) / \mu_{2} \rho_{2} \quad(\text { added })
$$

which is mathematically independent of the thickness of the material on which or within which it is located. However, the mass attenuation coefficient $\mu_{2}$ depends upon the radiation energy, which in turn must be selected to penetrate the first material. Therefore, there is a dependence upon the thickness of the primary or matrix material.

If the second material is substituted for the first rather than added to it, the thickness $\mathrm{x}_{1}$ is reduced to $\mathrm{x}_{1}-\mathrm{x}_{2}$ and equation 2 becomes

$$
\left(\mathrm{I} / \mathrm{Io}_{2}=\exp \left(-\mu_{1} \rho_{1}\left(\mathrm{x}_{1}-\mathrm{x}_{2}\right)\right) \times \exp \left(-\mu_{2} \rho_{2} \mathrm{x}_{2}\right)=(1 \pm f) \times(\mathrm{I} / \mathrm{Io})_{1}\right.
$$

From equation 4 it follows that

$$
x_{2}=-\ln (1 \pm f) /\left(\mu_{2} \rho_{2}-\mu_{1} \rho_{1}\right) . \quad \text { (substituted) }
$$

The ability to detect one material substituted for another depends upon how different they are, in absorption and/or density, as seen by what happens to the thickness $\mathrm{x}_{2}$ when the denominator in equation 5 approaches zero. 
In order to calculate the detectable amounts of toxic materials, we first find the optimum energy for the matrix material and its thickness, and then determine the thickness of added or substituted material using equation 3 or 5. The optimum energy is given by the "radiographers' rule of thumb" as being when the product $\mu \rho \mathrm{x}=2$. This rule of thumb is based upon signal-tonoise considerations [RWR85]. A program was written to search through the absorption coefficients for the matrix and thickness, finding the energy where $\mu \rho x=2$, and then calculate the thickness of added or substituted material which gives $1 \%$ contrast (equations 3 and $5, f=.01$ ) at that energy. Once the procedure for calculations is set up, it is easy to sweep through the periodic table and determine the detectable amount of all elements. The results are given graphically for a range of material thicknesses in Figures 13-15.

About $0.10 \mathrm{mg} / \mathrm{cm}^{2}$ of heavy metals such as lead (atomic number $=82$ ) can be found mixed in with $2 \mathrm{~cm}$ of light materials such as plastic (Figure 13). If the material is more like aluminum, the detection limit increases to the $\mathrm{mg} / \mathrm{cm}^{2}$ level (Figure 14). If the material is steel, the detection limit jumps to the $10 \mathrm{mg} / \mathrm{cm}^{2}$ level (Figure 15). These increases of detection limits with increasing atomic number of the matrix are due to the decreased contrast that results from needing higher energy radiation to penetrate the matrix. For instance, the energy increases from about $15 \mathrm{keV}$ for 2 $\mathrm{cm}$ of plastic, to $60 \mathrm{keV}$ for $2 \mathrm{~cm}$ of aluminum, to $240 \mathrm{keV}$ for $2 \mathrm{~cm}$ of steel. As energies increase, a thin coating of material becomes more and more transparent and therefore cannot be detected.

Are the calculated detection limits correct, or at least reasonable? To find out, we made digital radiographs of penetrameters made of beryllium, iron, and lead which were fastened to Lucite and to aluminum. Digital radiographs are shown in Figures 16 and 17. We take as a minimum detection limit that thickness of a particular material which gives absorbance equal to three times the "noise" level (random variation standard deviation, $\sigma$ ) in the signal for the matrix material. For Lucite and the experimental conditions for the radiograph, the $3 \sigma$ level occurred at a contrast of $3.4 \%$; for aluminum, the $3 \sigma$ level occurred at $1.6 \%$ contrast. The noise levels can easily be reduced by averaging multiple frames. We desire to compare the measured detection limits with calculated ones. Instead of calculating the absorbance at a particular energy as in the previous figures, it was necessary to calculate the effective absorbance for the spectrum of energies emitted by the $\mathrm{X}$-ray source. The source spectrum $S(E)$ was first calculated [TPR85] (Figure 18). The energy deposited in the detector was then calculated, for both the unattenuated and attenuated beams:

$$
\begin{aligned}
& I_{0} \equiv \int S(E) \cdot E \cdot d E \\
& I=\int S(E) \cdot E \cdot \exp (-\mu(E) \rho x) \cdot d E
\end{aligned}
$$

where $\mu(E)$ explicitly shows the energy dependence of the mass attenuation coefficient on energy. The effective mass absorption coefficient is then defined in the usual way:

$$
\mu_{\mathrm{eff}}=-\ln \left(\mathrm{I} / \mathrm{Io}_{\mathrm{o}}\right) /(\rho \mathrm{x})
$$

We calculated the amount of material for each element in the periodic table which yields the measured contrast at the $3 \sigma$ level. The calculated and measured detection limits are compared in Figure 19. This figure clearly demonstrates that radiographic detection limits for any material can be calculated with sufficient accuracy for planning needs. 


\section{Energy Dispersive X-Ray Fluorescence (XRF):}

The theoretical underpinning for estimating $\mathrm{x}$-ray fluorescence detection limits is not as firm as for radiography, at least without recourse to a good model for detector response. Such a model is beyond the scope of this work. We simply desire order of magnitude and relative estimates to guide our thinking. Detection limits are set by signal to noise ratios at the characteristic lines being measured. The "noise" is due to statistical fluctuations in the background, which in turn is due largely to scattered radiation and the degraded pulses from it which contribute to the background at all lower energies. Our approach is to estimate fluorescence and scattered radiation intensities, and to scale these calculated values with experimental data if necessary.

Estimates are based upon considerations depicted in Figure 20. A beam of x-ray of intensity Io impinges on a substance at angle $\psi_{1}$, is attenuated as it passes into the material to a depth $\mathrm{x}$, where it interacts in the infinitesimal thickness $\mathrm{dx}$ by scattering or producing fluorescence after photoelectric absorption, and then is attenuated again in passing to the detector at angle $\psi_{2}$ with intensity $\mathrm{I}$. The path length from the surface to $\mathrm{dx}$ and the attenuation along that path length are

$$
I_{1}=x / \sin \psi_{1},\left(I / I_{0}\right)_{1}=\exp \left(-\mu_{1} \rho l_{1}\right) .
$$

Likewise, the path length from $\mathrm{dx}$ to the surface is and the attenuation along that path are

$$
l_{2}=\mathrm{x} / \sin \psi_{2}, \quad\left(\mathrm{I} / \mathrm{I}_{0}\right)_{2}=\exp \left(-\mu_{2} \rho \mathrm{l}_{2}\right) .
$$

Whatever the event that occurs within $\mathrm{dx}$ (absorption, scatter, or fluorescence), the conversion is proportional to the incident radiation at that point and is proportional to some conversion factor or product of factors (cross section, fluorescence yield, branching factor). Let this generalized factor be given by $e$. The change in intensity at $d x$ is given by

$$
\mathrm{dI}=-\mathrm{I} \varepsilon \rho \mathrm{dI}_{1} \text {. }
$$

Ignoring geometric factors, the change observed at the surface is found by combining equations 7 , 8, and 9:

$$
\begin{aligned}
& \text { change }=(\text { attenuation in }) \times(\text { conversion }) \times(\text { attenuation out }) \\
& \mathrm{dI}=-\mathrm{I}_{0} \cdot \exp \left(-\mu_{1} \rho \mathrm{l}_{1}\right) \cdot \varepsilon \rho \mathrm{dl}_{1} \cdot \exp \left(-\mu_{2} \mathrm{\rho l}_{2}\right) .
\end{aligned}
$$

In integral form, this equation becomes

$$
I=-\operatorname{Io\varepsilon } \rho \int_{0}^{x} \exp \left(-\rho x\left(\mu_{1} / \sin \psi_{1}+\mu_{2} / \sin \psi_{2}\right) \cdot d x / \sin \psi_{1}\right.
$$

When integrated to thickness $\mathrm{x}$, the intensity becomes

$$
\left(1-\exp \left(-\rho x\left(\mu_{1} / \sin \psi_{1}+\mu_{2} / \sin \psi_{2}\right)\right) \cdot \frac{\mathrm{I}_{0} \rho}{\sin \psi_{1} \rho\left(\mu_{1} / \sin \psi_{1}+\mu_{2} / \sin \psi_{2}\right)}\right.
$$


We are dealing with two limiting cases, namely "infinitely" thin when fluorescing a thin layer, and "infinitely" thick when scattering from the substrate on which the fluorescer is coated. For the thin fluorescer, equation 12 becomes

$$
\begin{aligned}
& \mathrm{I}_{\mathrm{f}}=\mathrm{I}_{0} \varepsilon \rho \mathrm{x} / \sin \psi_{1} \\
& \varepsilon=\mathrm{C}_{\mathrm{A}} \tau_{\mathrm{A}} \mathrm{w}_{\mathrm{A}} \mathrm{g}_{\mathrm{L}}
\end{aligned}
$$

where the conversion constant is a set of physical constants (weight fraction of the element of interest, and its photoelectric mass attenuation coefficient, fluorescent yield, and branching ratio). For a thick scatterer, equation 12 becomes

$$
\begin{aligned}
& I_{s}=\frac{I_{0} \varepsilon}{\mu_{1}+\mu_{2}\left(\sin \psi_{1} / \sin \psi_{2}\right)} \text { (thick scatterer) } \\
& \varepsilon=\sigma_{C}+s_{R}=\sigma
\end{aligned}
$$

where the conversion constant is the sum of the Compton and Rayleigh mass attenuation (scatter) coefficients.

Equations 14 and 15 are the tools we need to proceed. Detection limit data for thin films can be found in the literature [VGM93]. The detection limits are functions of sensitivity and the background. Equations 14 gives the shape of the sensitivity curve (response per amount as a function of atomic number). Knowing this, we can estimate the contribution of the background. That is,

$$
\mathrm{mdl}_{\mathrm{t}}=3(\mathrm{~B} / \mathrm{t})^{1 / 2} \cdot \mathrm{K}
$$

where $\mathrm{mdl}_{\mathrm{t}}$ is the minimum detection limit in time $\mathrm{t}, \mathrm{B}$ is the background count rate, and $\mathrm{K}$ is the sensitivity factor ( $\mathrm{g} / \mathrm{cm}^{2} / \mathrm{cps}$ ) from equations 14 for each element. It is not possible to separate out the time (not specified in the reference) and the beam intensity Io. Estimates of Io were therefore made from the program TUBDET [TPR85] and from "zero background" detection limits where only counting statistics for the signals themselves are relevant. Once each background rate is estimated for each element from equation 16, the functional relationship between background and atomic number can be found. These relationships are shown in Figure 21. Using equation 14 with $\mathrm{e}=\mathrm{s}$, the fraction of the scattered intensity which results in background at any energy (or element) is determined. Then equation 14 is used again to estimate the background for any thick scatterer. For our illustrative purposes, we select plastic, aluminum, and steel. Knowing the background and sensitivity factors, the minimum detection limits may be calculated for each element (equation 16). The results are shown in Figure 22. All the heavier elements can be detected at the sub-microgram per square centimeter level on the surface of any substance (in the absence of spectral interferences).

We went into the laboratory and measured detection limits. The system used is not as ideal as that used to make the estimates above. Instead of the quasi-monochromatic excitation source above, a hardened beam from a silver $x$-ray tube, filtered by $.013 \mathrm{~cm}$ tin, was used. This will inherently produce more background. The system is not engineered, but rather is the collection of components placed together to produce and detect $\mathrm{x}$-ray fluorescence. These things being said, the results are still rather good. All the heavier elements can be detected at sub-microgram per square centimeter level (Figure 23). The ratio of measured to estimated detection limits are shown in Figure 24. The measured values are about 6 to 20 times higher. The off-set of the measured values is probably both due to assumptions in the estimates and to non-optimization of the measurement 
system. Figure 24 may indicate the magnitude of enhanced performance of a good, commercial instrument over a quickly assembled system.

Summary of Radiography and X-Ray Fluorescence Detection Limits:

Detection limits for $\mathrm{x}$-ray radiography and fluorescence have been estimated and measured. There is excellent agreement between radiographic measurements and estimates. There is reasonable agreement between fluorescence measurements and estimates, particularly given the differences in experimental arrangements and some of the hand-waving in the estimates (detector response function). Radiographic detection limits below a milligram per square centimeter are obtainable for heavy elements, but may be much higher if the substrate materials are thick and/or composed of heavy materials. Fluorescence detection limits, on the surfaces of materials, are less than one microgram per square centimeter for most materials, and may be an order of magnitude less in a carefully engineered system.

Documentation

The MWO characterization project was documented in three different ways: written reports, poster presentation, and Mosaic multimedia presentation. The poster and Mosaic presentations are discussed here.

\section{Poster Presentation}

A poster presentation was developed for the MWO demonstrations and held at the end of the fiscal year. The poster session is miniaturized and is shown in Figures $25 \& 26$. The poster presents the characterization system design for the MWO project. The associated technologies are explained and displayed on separate panels around the system block diagram of the characterization system as shown in Figure 25. A selected group of central research developed during this project are displayed on panels in Figure 26. Three major research results are displayed in this portion of the poster presentation, material identification and volume determination, technology integration, and minimum detectable limits. The poster is available for DOE demonstration.

\section{Mosaic Presentation}

The Mosaic program was used to document a portion of the FY94 MWO project. Mosaic is a networked information discovery, retrieval, and collaboration tool and World Wide Web browser (explained below). Mosaic is a product of the Software Development Group of the National Center for Supercomputing Applications (NCSA) at the University of Illinois at Urbana-Champaign. NCSA Mosaic is copyrighted but free for academic and research use. Copies of NCSA Mosaic can be retrieved in both source and executable binary form from NCSA's anonymous FTP server (ftp.ncsa.uiuc.edu).

The World Wide Web (W3) is a universe of network-accessible information, a world wide encyclopedia. It is an initiative started at CERN (European Laboratory for Particle Physics located in Geneva, Switzerland), now with many participants. It has a body of software, and a set of protocols and conventions. W3 uses "hypertext" and multi-media techniques to make the web easy for anyone to roam, to browse, and to contribute. Future evolution of $W 3$ is coordinated by the W3 Organization.

Mosaic was used to document the MWO project for three reasons; it is an expandable multimedia documentation tool, it is an excellent marketing tool, and it can be used for data sharing. Mosaic is an excellent multi-media documentation tool that can be expanded upon as the MWO 
project progresses. The Mosaic document can be linked to and from other Mosaic documents. For example, the LLNL MWO project document can be linked to a document in Washington. The link is accomplished over the network so the LLNL document does not need to reside on the computer in Washington. Mosaic documents can be viewed across different computer platforms (i.e. Macintosh, IBM, Sun and SGI computers). Mosaic encompasses many different standard viewing tools for displaying graphics, photographs, animations, and movies.

Mosaic is also a marketing tool. The World Wide Web used by the Mosaic program provides access to the MWO document for all interested organizations around the world. The Mosaic program also provides an easy to use data retrieval tool. For example, if a technician at the Idaho National Engineering Laboratory (INEL) needs to compare radiographs of waste objects acquired there to radiographs of similar work done at LLNL, he simply hunts for the information and pushes a graphic button on the computer screen with the mouse and the data is automatically downloaded. The technician does not need to be an expert in computer operating systems and Internet software and hardware to perform this task.

Figure 27 shows the introduction page for the MWO project as displayed by the Mosaic program. A skeleton was produced for the entire MWO project; however, only the characterization portion of the document was developed. The other MWO functional areas will be filled in at a later date, depending on continued funding. The MWO Mosaic document must be reviewed and released before it is placed in the LLNL home page and available for public viewing.

\section{SUMMARY}

We reviewed the program objectives for this year's project, and pertinent regulations. Based upon this review and an assessment of the non-intrusive inspection and characterization methods available to us at LLNL, we developed a characterization systematic plan to satisfy all requirements. The output of the characterization system is either a treatable object, non-treatable object, object to be further singulated, or a clean object that need not be treated. The characterization system is also designed to inspect objects after treatment to ascertain if treatment is complete. The characterization methods used this year were radiography, tomography, gamma ray spectroscopy, and x-ray fluorescence.

We have characterized selected mock waste items. We are able to unambiguously identify hazardous inorganic materials on the surface of objects. We can also ascertain the presence of heavy metals within objects. We can determine volumes (computed tomography) and surface areal densities ( $\mathrm{x}$-ray fluorescence) to within a few percent. We have calculated and confirmed minimum detectable amounts of materials. These detection limits depend upon everything else that is present, but range down to sub-milligram per square centimeter levels for heavy elements by radiographic and tomographic methods, and below the microgram per square centimeter on the surface by $x$-ray fluorescence. We have demonstrated the efficiency possible by integrating radiographic with tomographic data. Here, we developed a technique to only use radiographic data where the material is homogeneous (fast), and then switch to tomography in those areas where heterogeneity is detected (slower). This work is documented in Mosaic, an Internet multi-media browser. This document is used to demonstrate the ability to share data and information world-wide.

Beyond the scope of this year's work are some issues for a functioning mixed waste treatment facility. Sampling and chemical analysis would be required, particularly for organic materials and gases which might be hazardous. The characterization methods described here would most effectively be employed in the work area and treatment cell itself. Ideally, the characterization 
would be done robotically, and either automatically or semi-automatically in order to improve efficiency and safety. The benefits of automated characterization include:

- Improved safety due to reduced operator exposure to radiation and hazardous objects

- Improved accuracy for sorting and inspection techniques

- Improve control and documentation of sorting and treatment operations

- Reduced time involved in characterization of individual objects

- Reduced costs associated with treatment, recycling and disposal of waste.

\section{ACKNOWLEDGMENTS}

We thank Derrill Rikard and Earl Updike for help in setting up experiments and obtaining data. We also thank Marsha Bell for developing the art work presented in this paper, the poster presentation and the Mosaic presentation. This work was funded by the Office of Technology Development in DOE's Environmental Restoration and Waste Management Program and performed under the auspices of the U.S. Department of Energy by the Lawrence Livermore National Laboratory under Contract W-7405-Eng-48. 


\section{REFERENCES}

[BRN93] C. H. Brown, R. J. Gehrke, C. A. Langton, N. M. Askew, T. Kan, and W. E. Schwinkendorf, "Treatment Technonogy Analysis for Mixed Waste Containers and Debris," DOE/MWIP-20, Oak Ridge National Laboratory, Oak Ridge, Tennessee, November 1993.

[DEB88] K. Debretin and R. G. Helmer, Gamma- and X-Ray Spectrometry with Semiconductor Detectors, (Elsevier, New York, NY, 1988).

[GRA94] E. Grasz, E. Domning, D. Heggins, L. Huber, R. Hurd, H. Martz, P. Roberson, K. Wilhelmsen, "Summary of LLNL's Accomplishments for the FY93 Waste Processing Operations Program," Preapared for the U.S. Department of Energy, 031548002/D94-012.337m/V-1.4, Lawrence Livermore National Laboratory, Livermore, CA, March, 1994.

[KAW90] S. Kawasaki, M. Kondo, S. Izumi, and M. Kikuchi, "Radioactivity Measurement of Drum Package Waste by a Computed-Tomography Technique," Appl. Radiat. Isotopes 41, 983 (1990).

[MAR94] H. E. Martz, D. J. Schneberk, and G.P Roberson, "Real-Time Radiography, Digital Radiography, and Computed Tomography for Non intrusive Waste Drum Characterization," Proceedings of the Nondestructive Assay and Nondestructive Examination Waste Characterization Conference, Pocatello, Idaho, February 14-16, 1994; UCRL-JC-115670, Lawrence Livermore National Laboratory, Livermore, CA, February 1994.

[RE192] P. Reimers, "Quality Assurance of Radioactive Waste Packages by Computerized Tomography, Task 3, Characterization of Radioactive Waste Forms; A Series of Final Reports (1985-89) - No. 37," Nuclear Science and Technology, EUR 13879 EN, Commission of the European Communities, Luxembourg, 1992.

[ROB94] G. P. Roberson, H. E. Martz, D. J. Deckman, D. C. Camp, S. G. Azevedo and E. R. Keto, "Characterization of Waste Drums Using Nonintrusive Active and Passive Computed Tomography," Proceedings of the Nondestructive Assay \& Nondestructive Examination Waste Characterization Conference, Idaho State University, Pocatello, Idaho, February 14-16, 1994; UCRL-JC-118317, Lawrence Livermore National Laboratory, Livermore, CA, August, 1994.

[RWR85] Richard W. Ryon, "Optimum Ranges for X-Ray Thickness Measurements," LLNL Report UCID-20518, 1985.

[TPR85] X-ray tube spectrum calculated from a modified version of a subroutine in the National Institutes of Standards and Technology program known as NBSCGS, NIST Technical Note 1213, by Tao, Pella, and Rousseau). Program adapted to run on a MacIntosh by Richard Ryon.

[VGM93] "Handbook of X-Ray Spectrometry," Rene Van Grieken and Andrzey Markowicz, editors, Marcell Dekker, 1993, p. 178. 


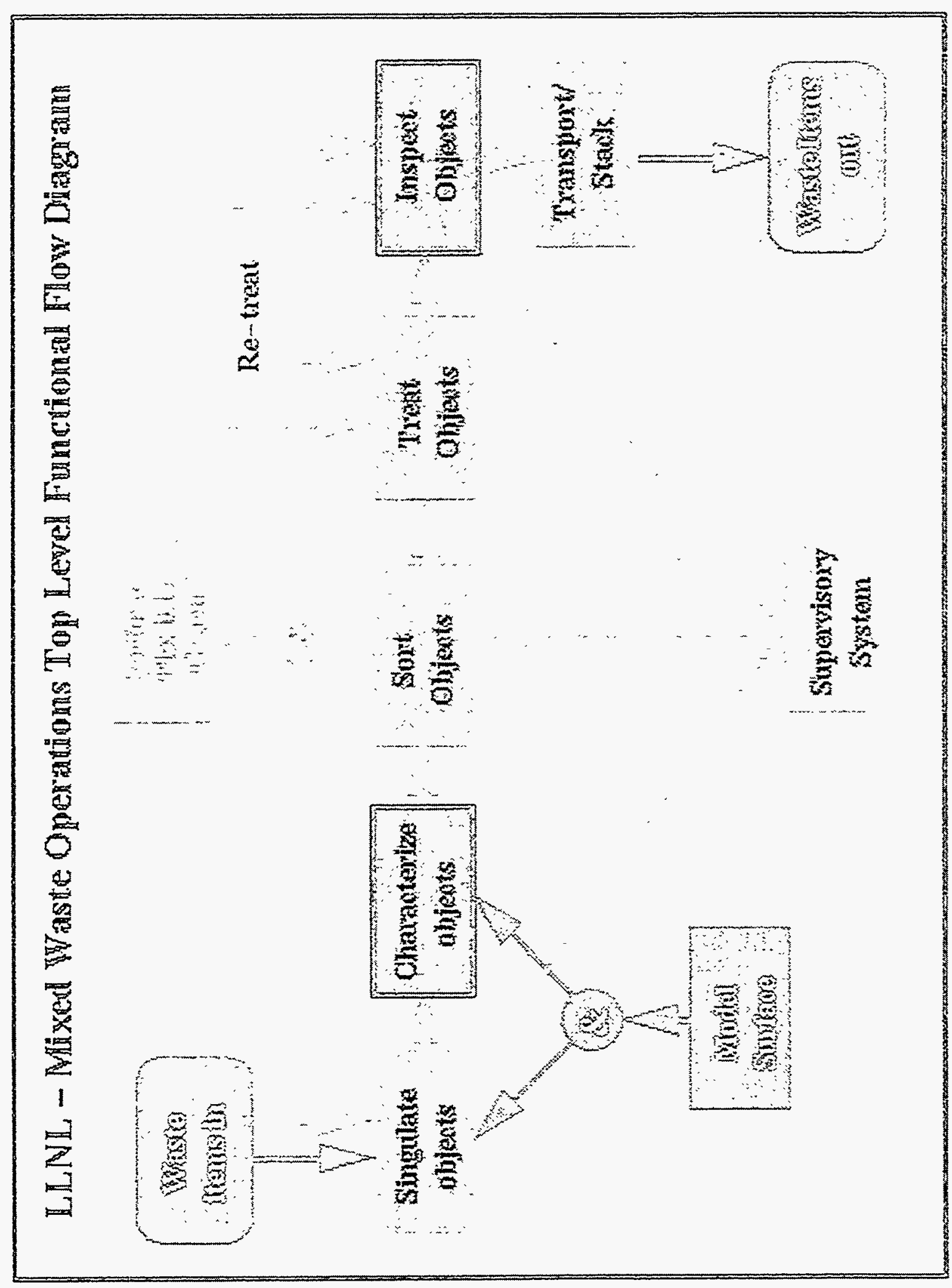

ఏ 

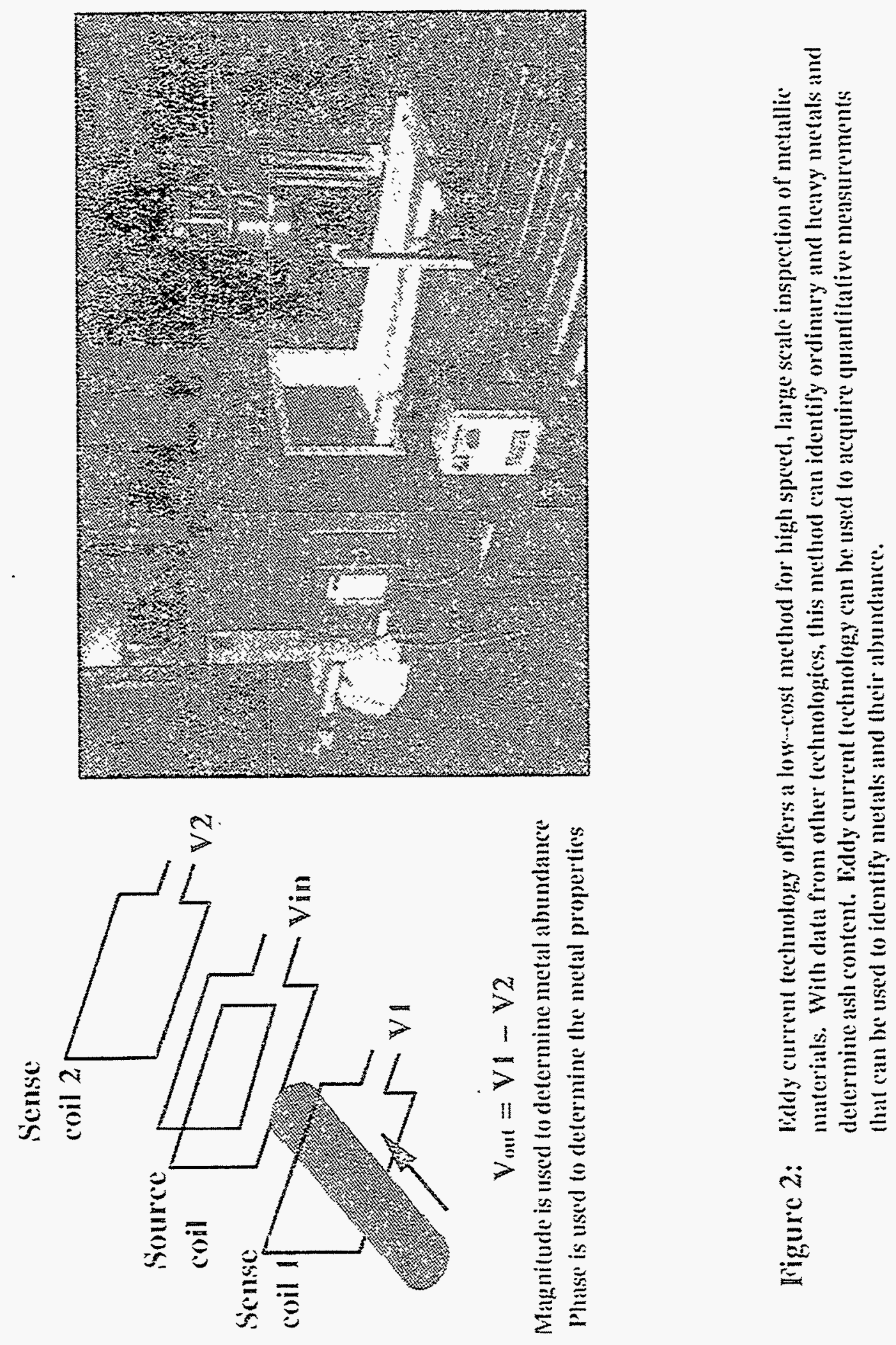

$\underset{E}{0}$ 

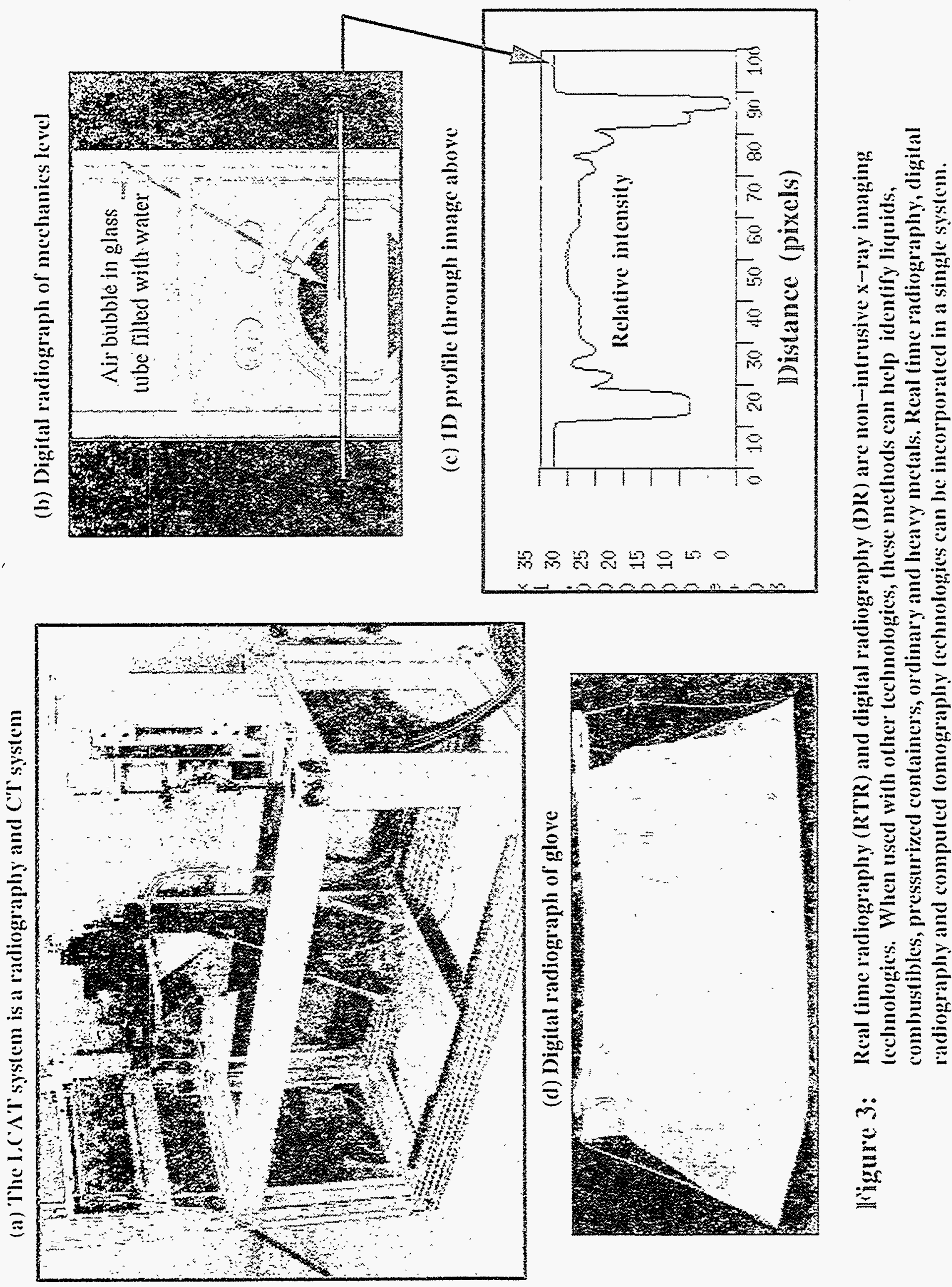

0
$=$
$=$
$=$ 


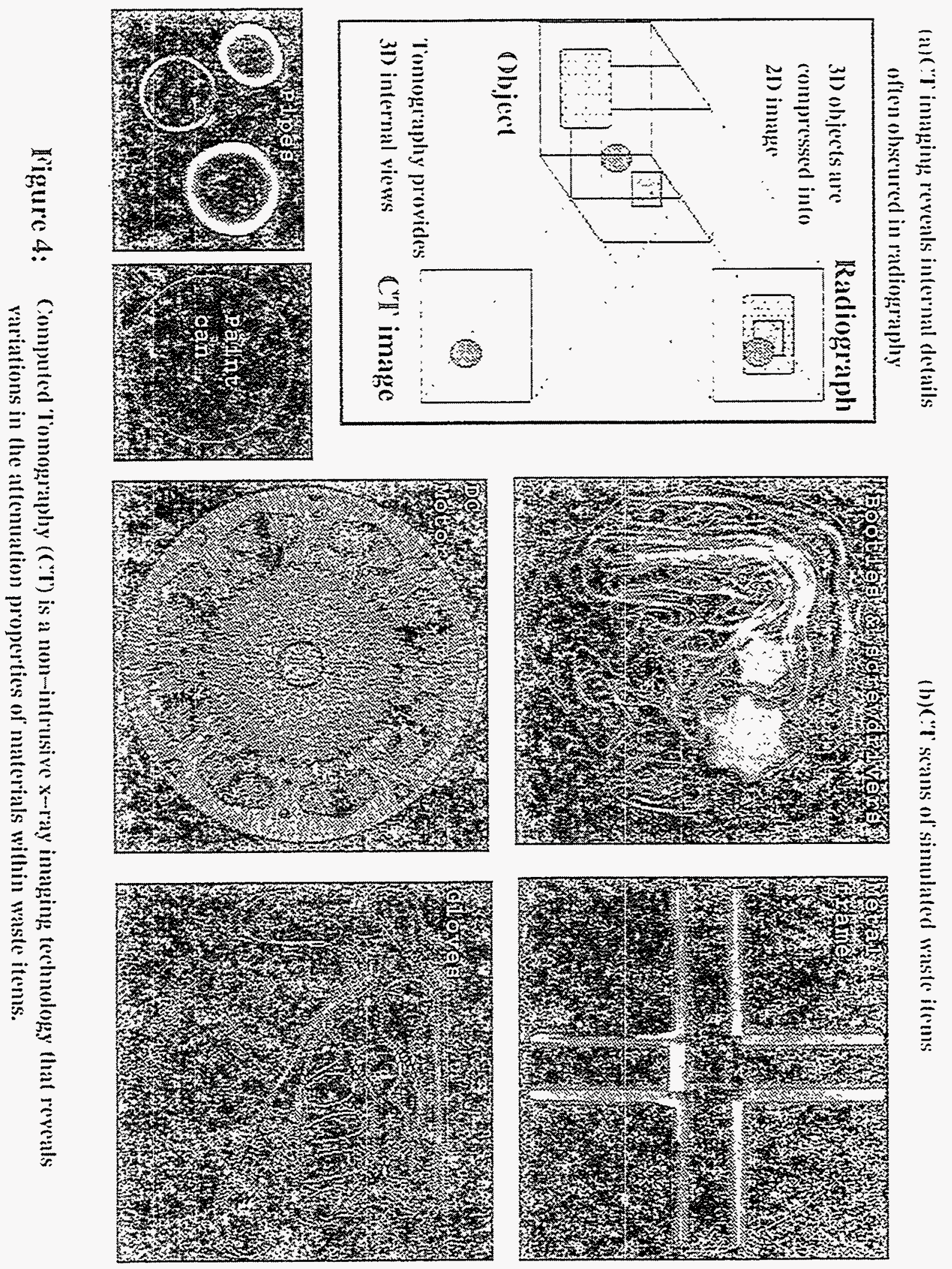



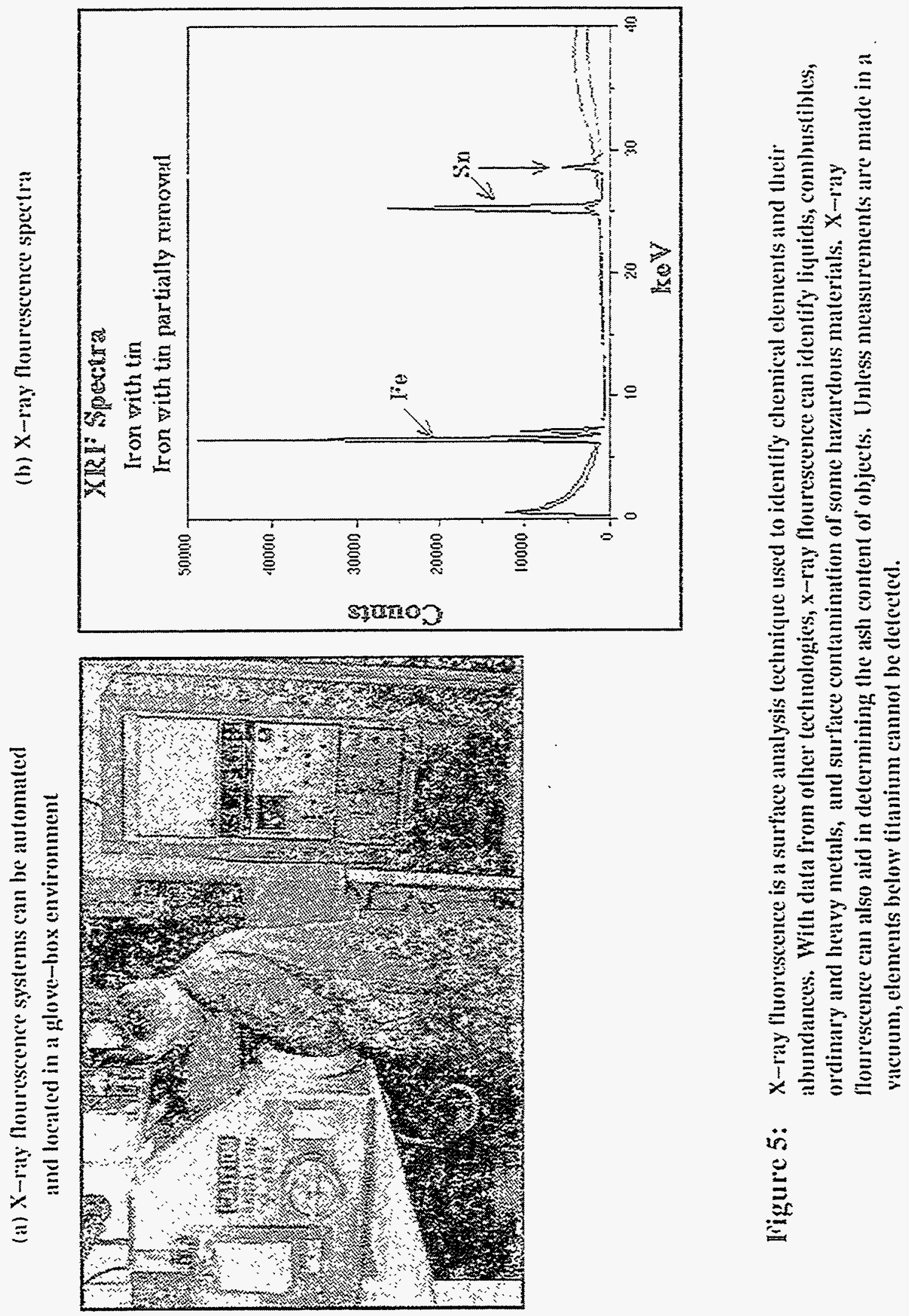

$\underset{D}{0}$ 


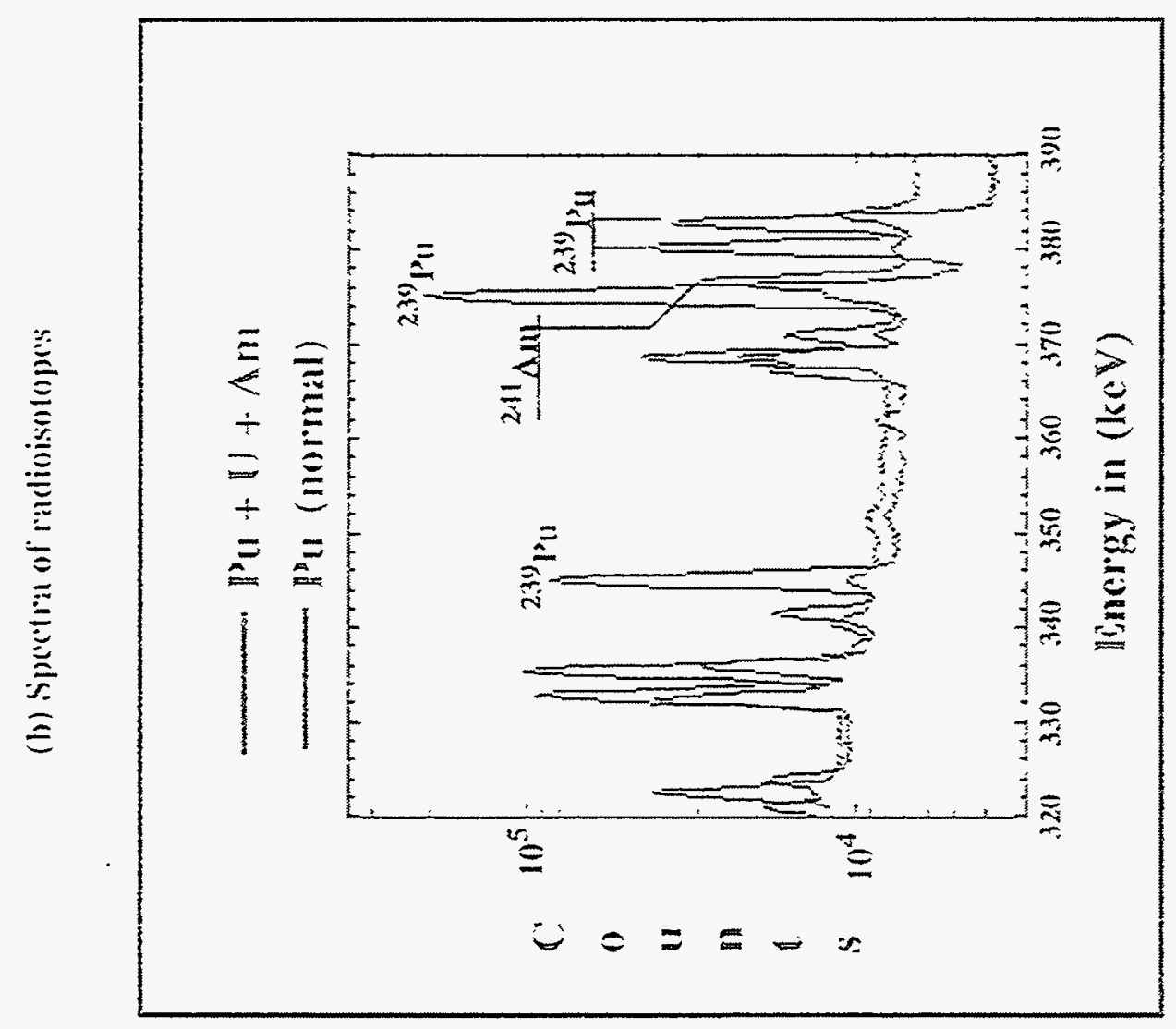

方 

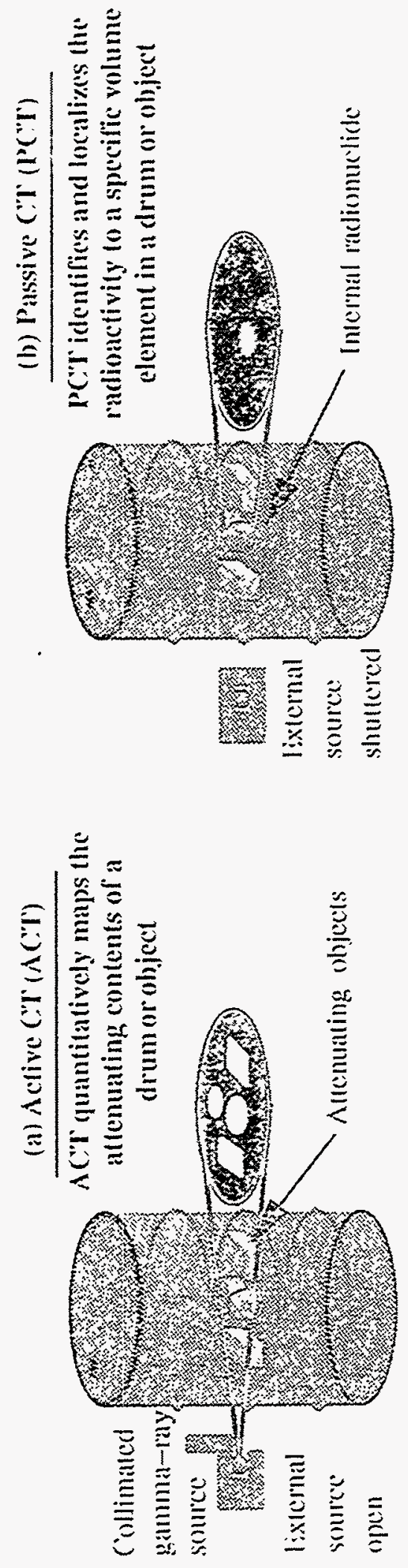
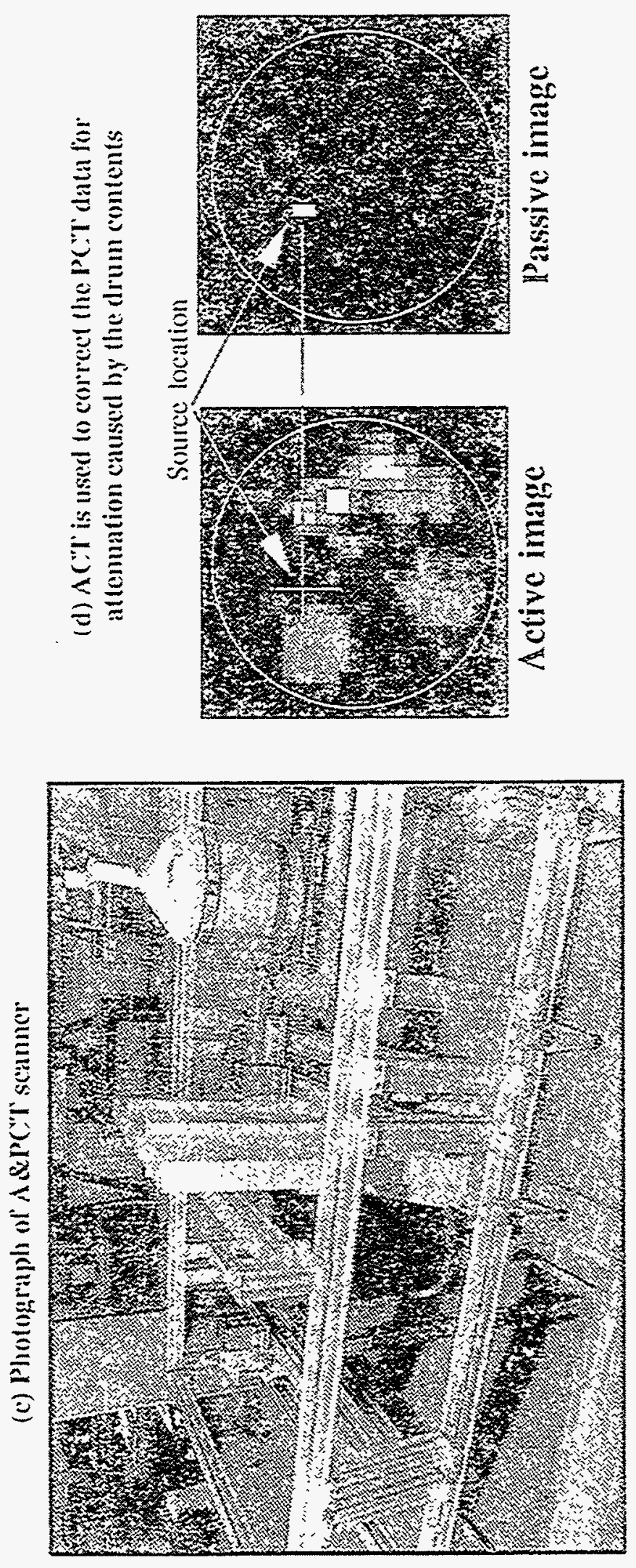
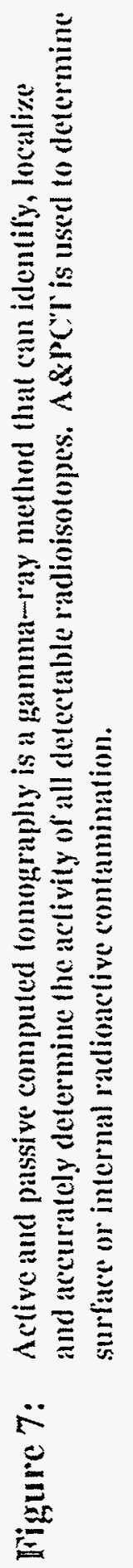
Ke:
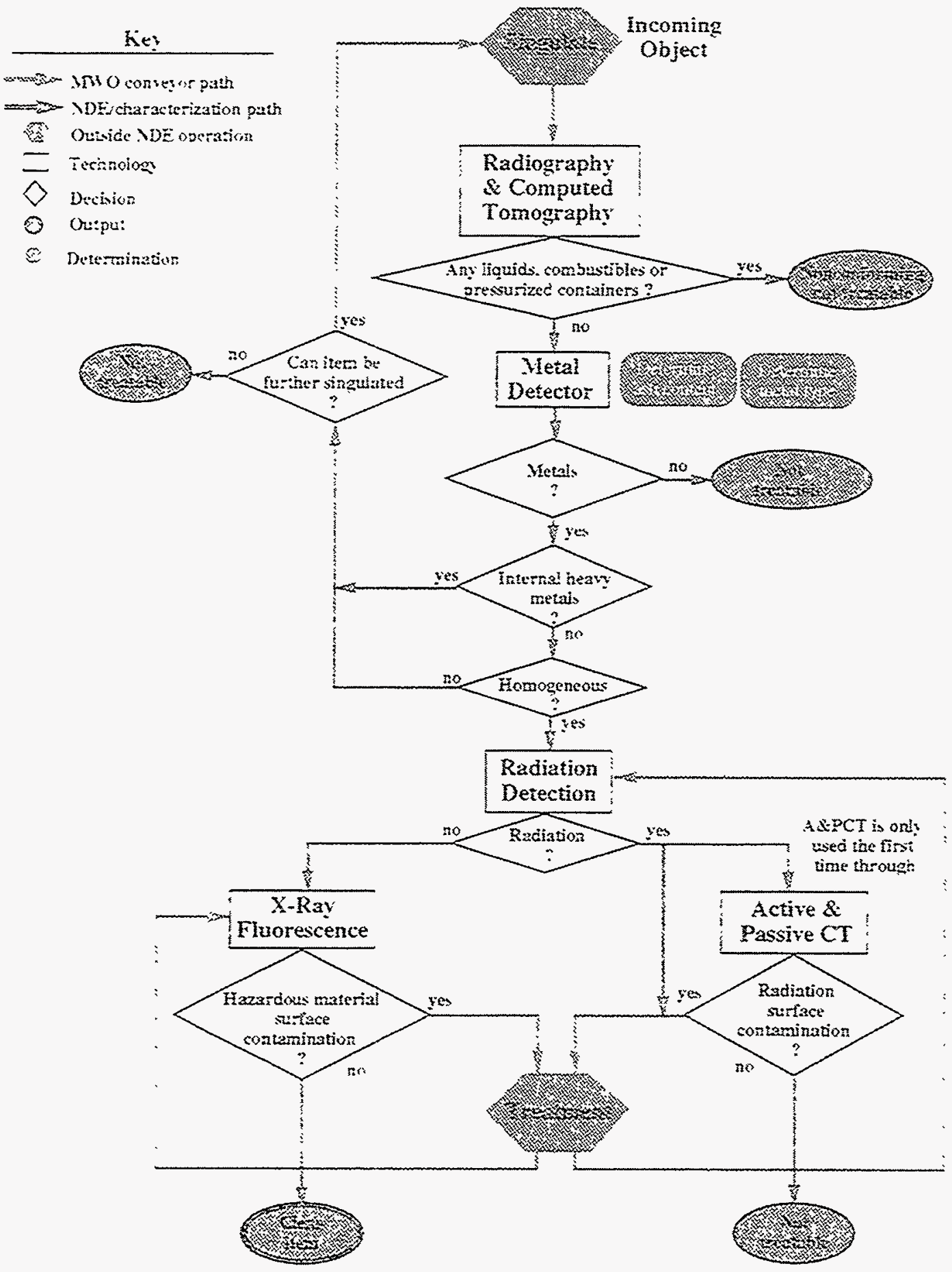

Figure 8: The MWO characterization system design includes six basic $Y D E N D A$ technologies: radiography, computed tomography, metal detection, radiation detection. active and passive computed tomography and $\mathrm{x}-\mathrm{ray}$ fiuorescence 

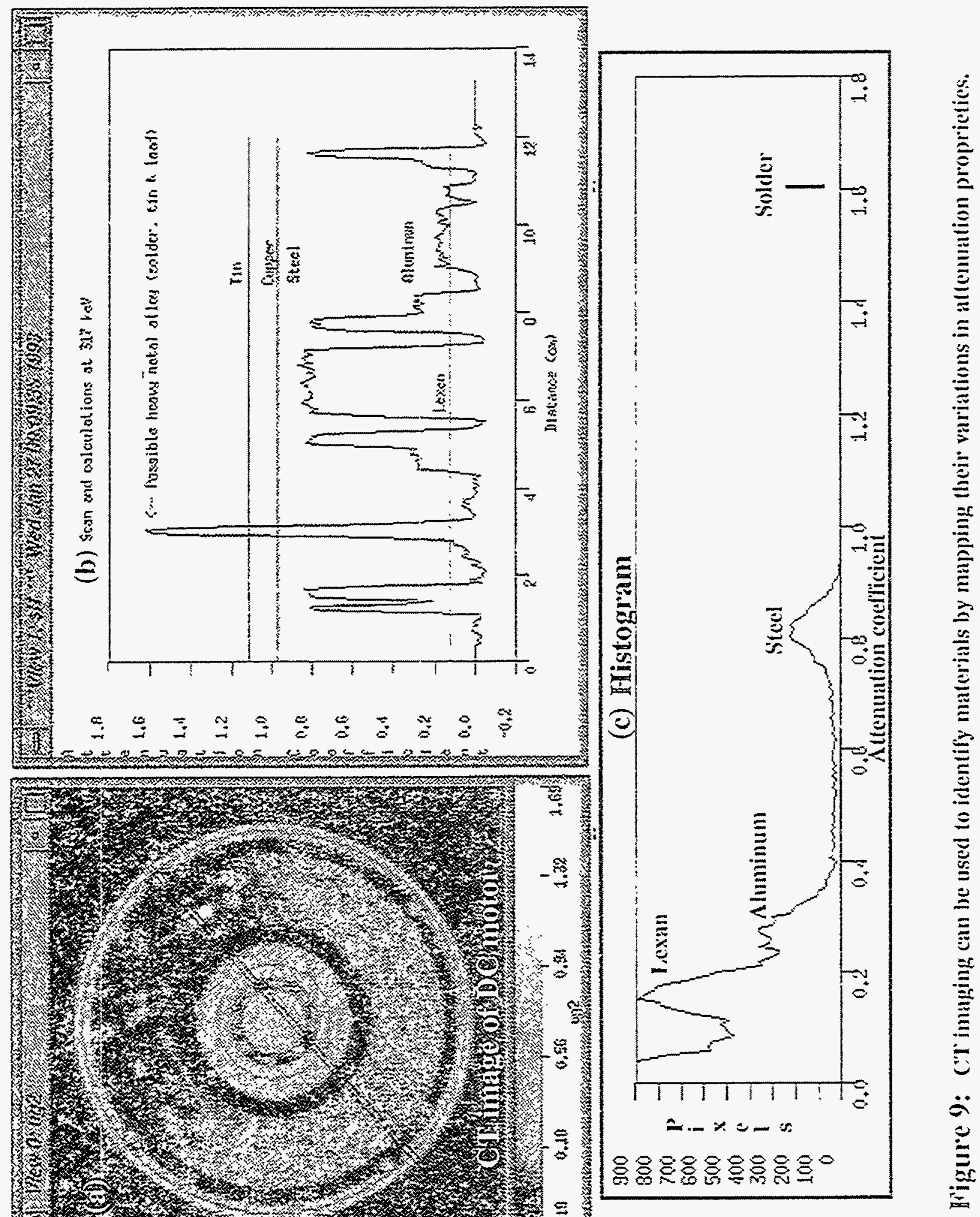

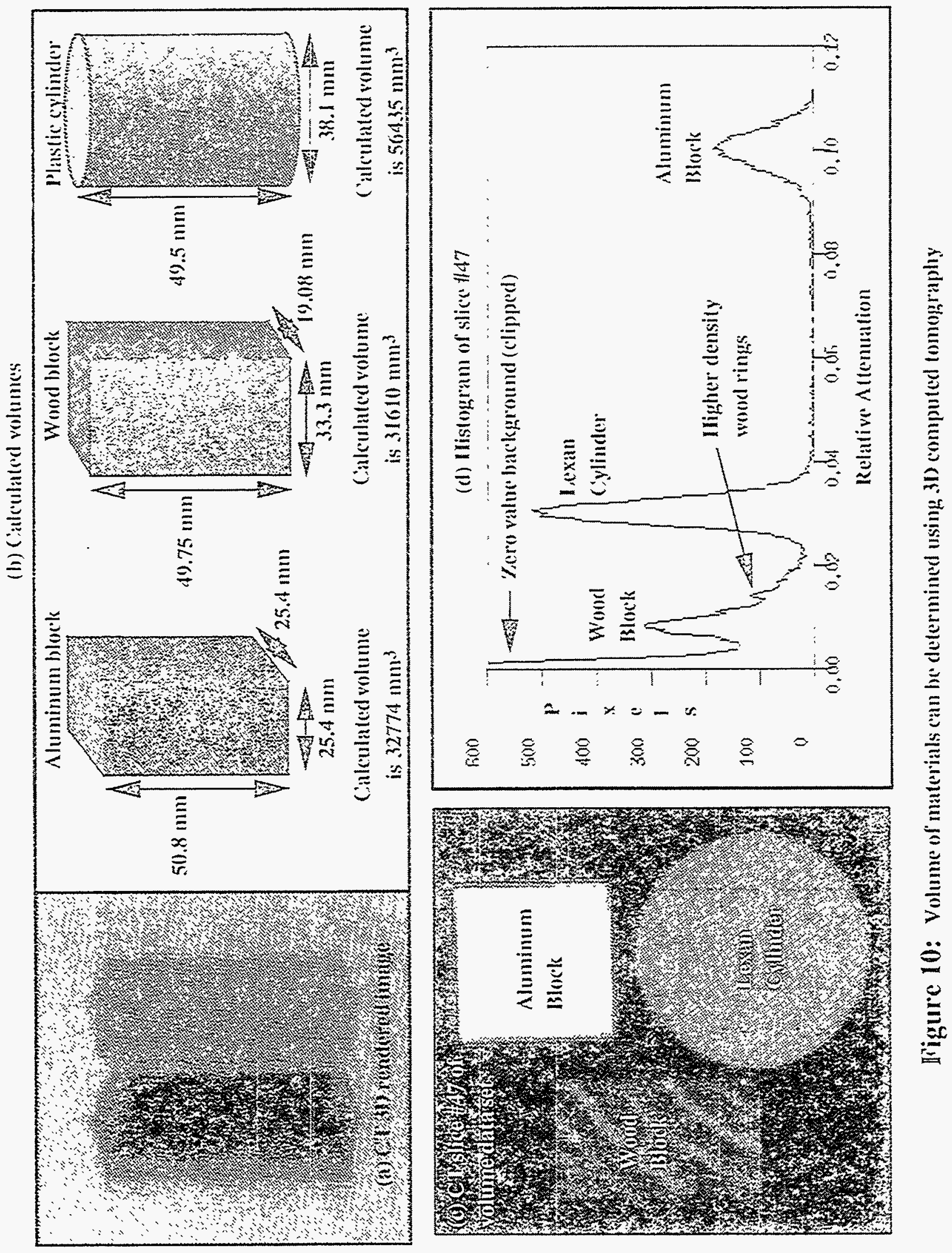

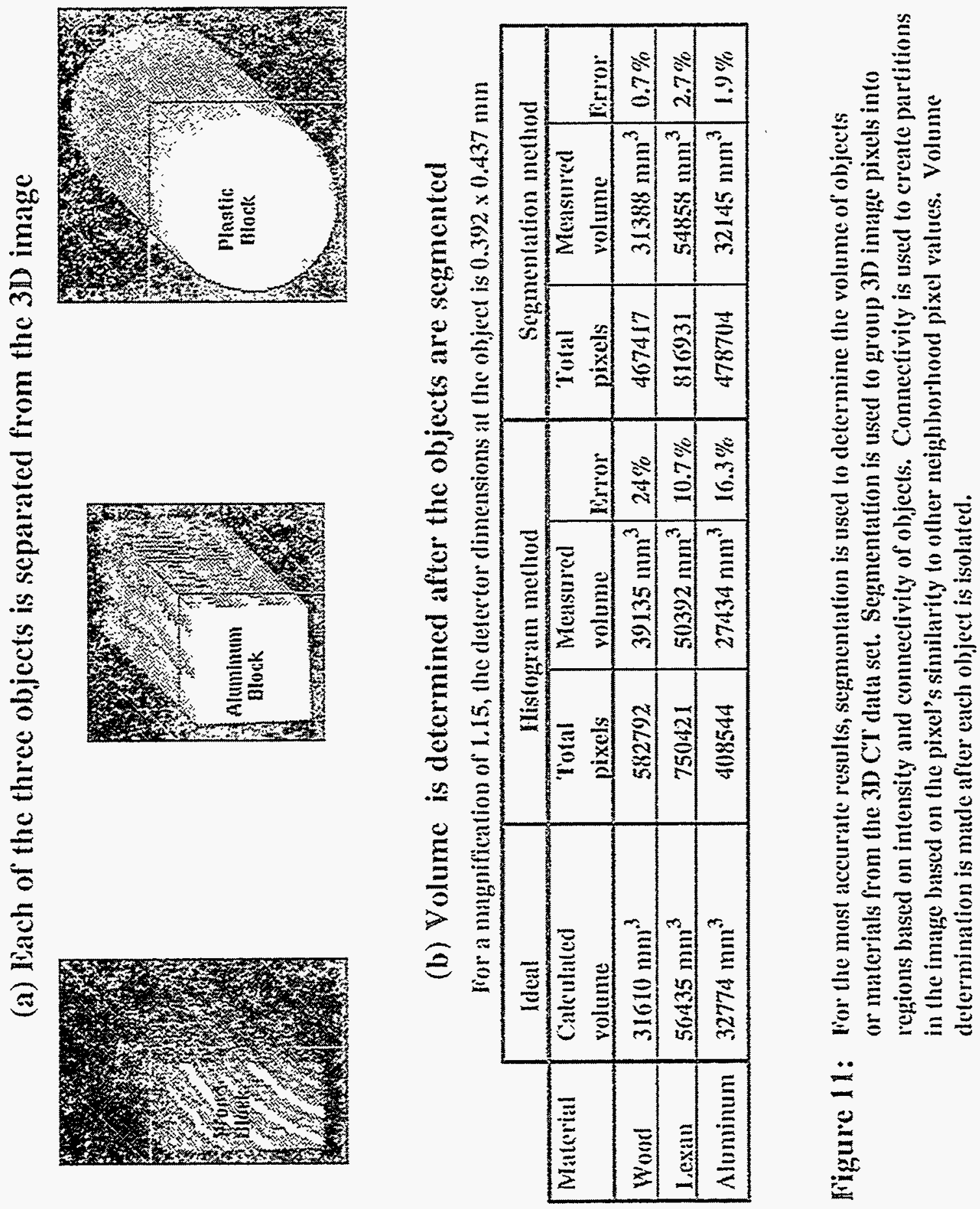


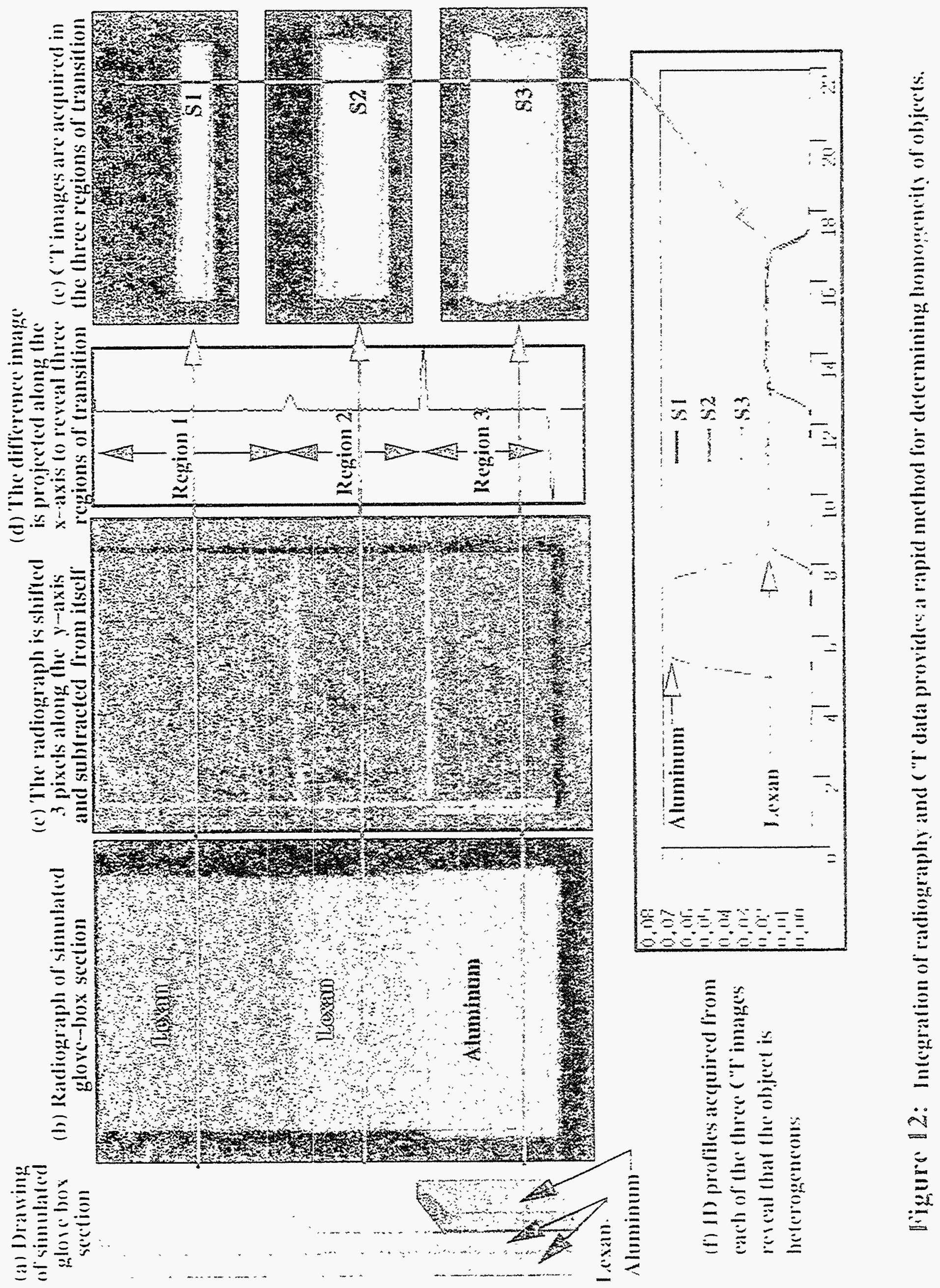




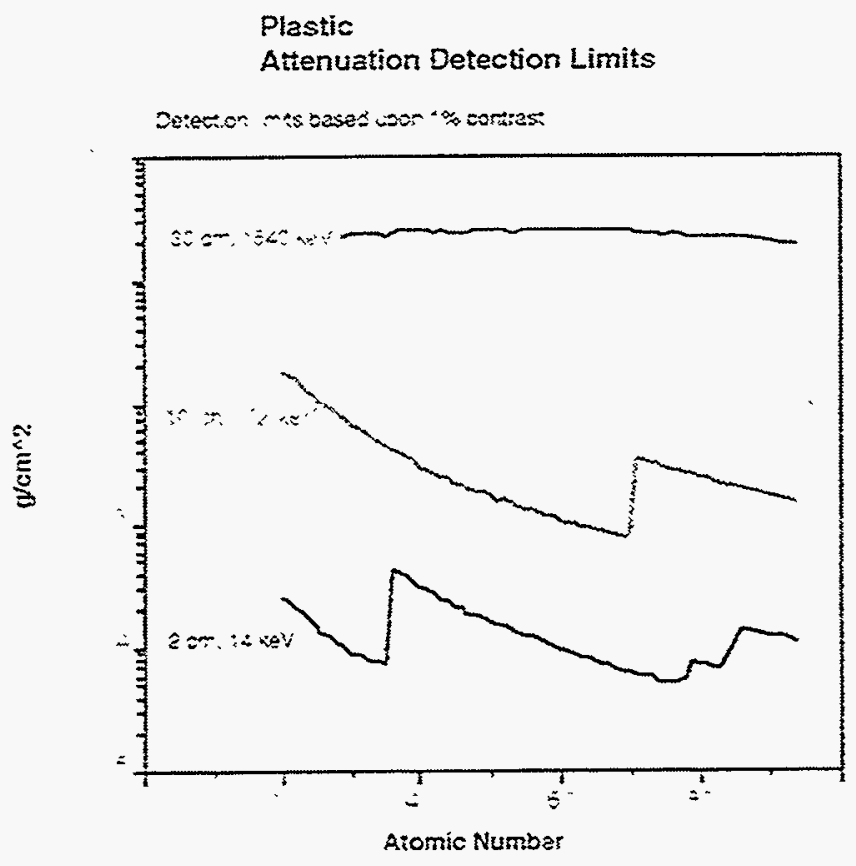

Figure 13. Calculated radiographic minimum detectable amounts of elements adided to plastic of selected thicknesses

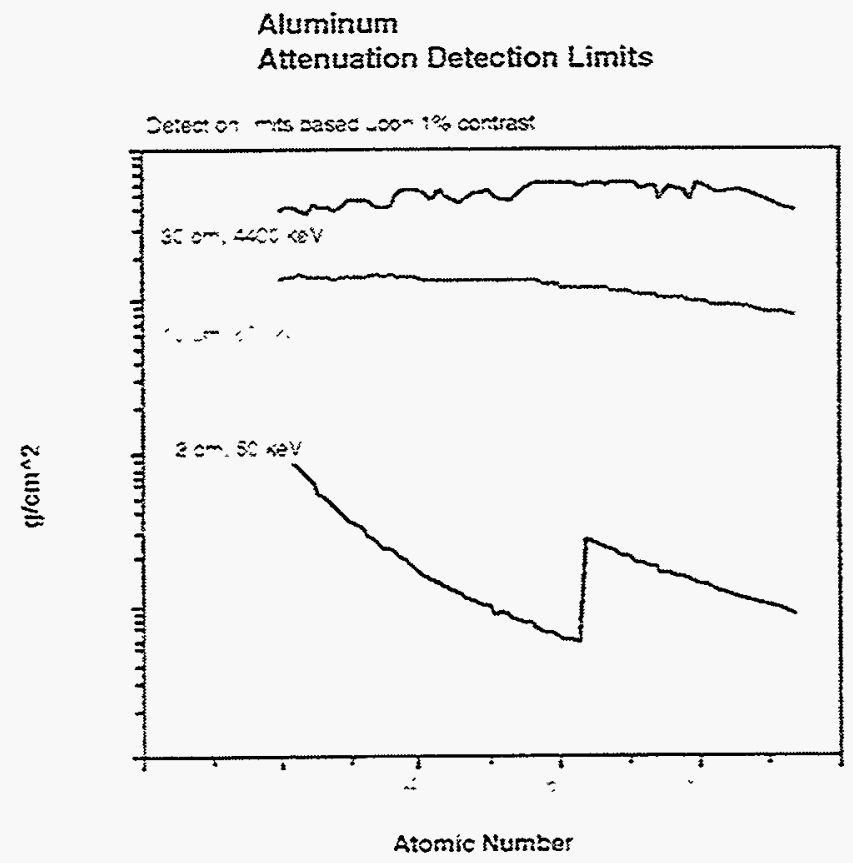

Figure 14. Cakulated radiographic minimum detectable amounts of elements added to aluminum of selected thicknesses 


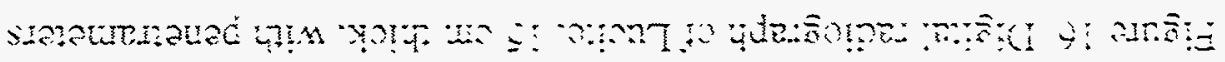

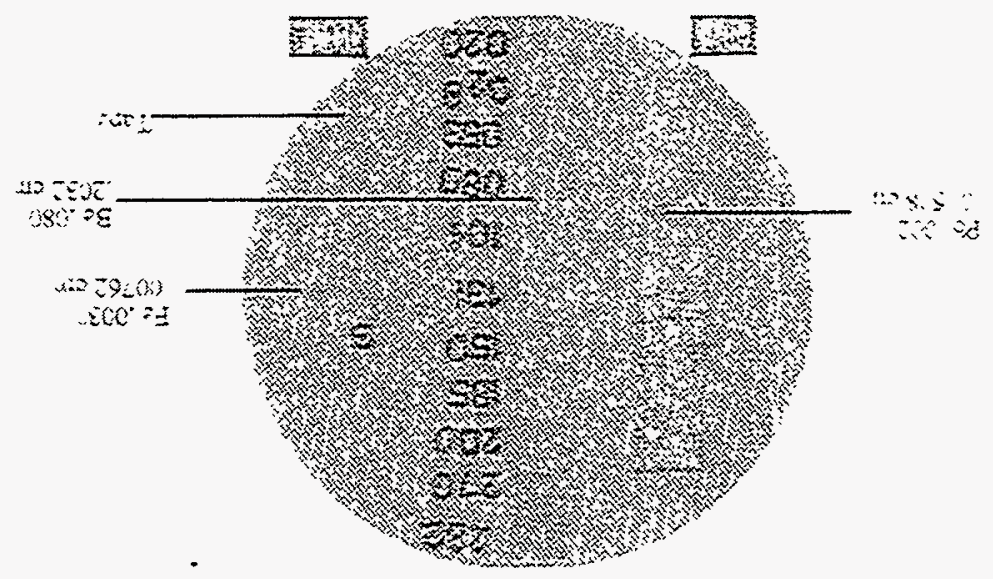

sasseryolut pamoatas

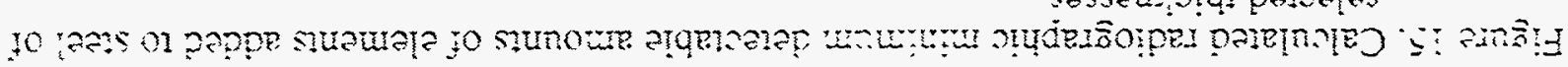

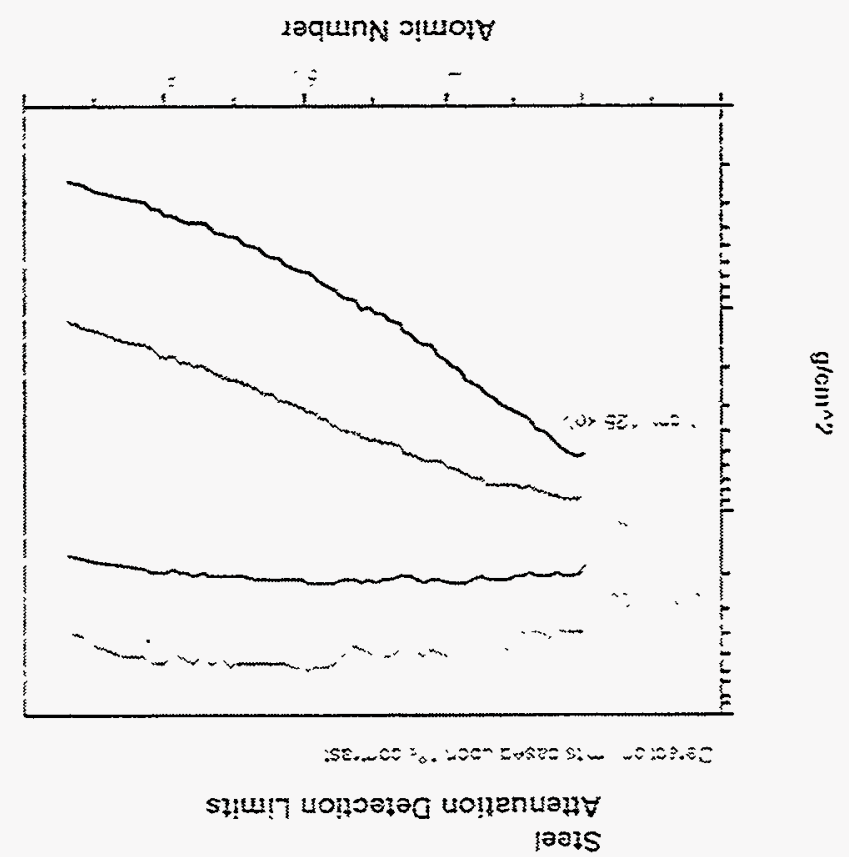




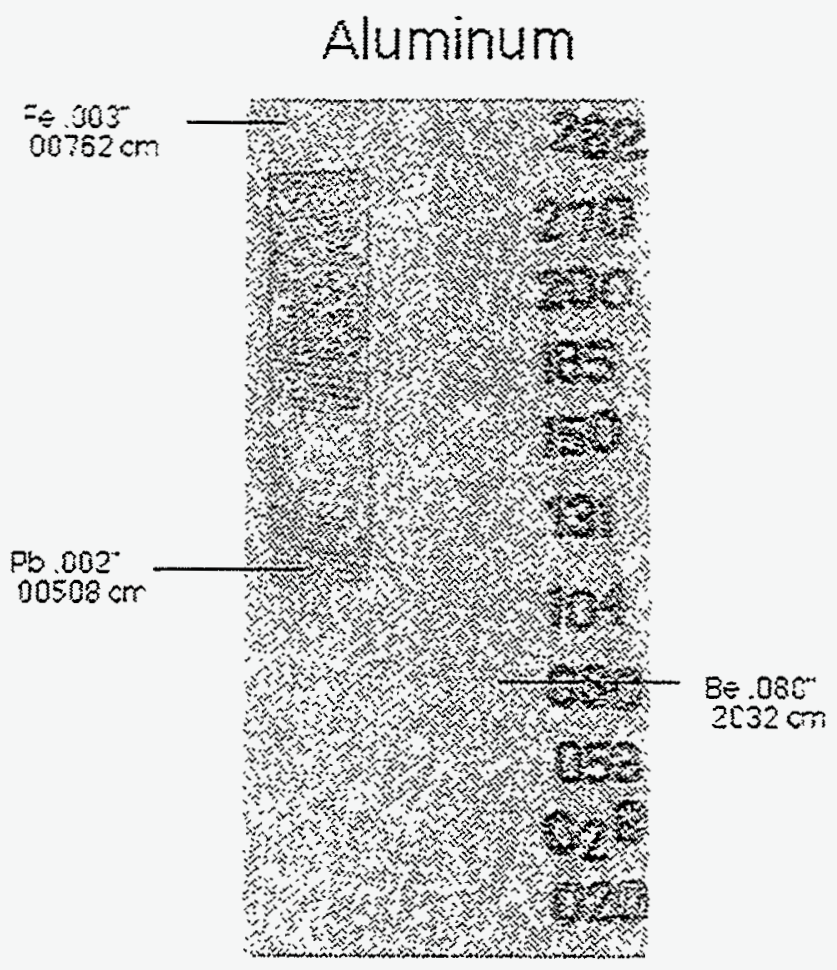

Figure 17. Digital radiograph of aluminum. $2.5 \mathrm{~cm}$ thick. with penetrameters

W tube, $160 \mathrm{kV}$ with $0.40 \mathrm{~mm}$ Cu filter

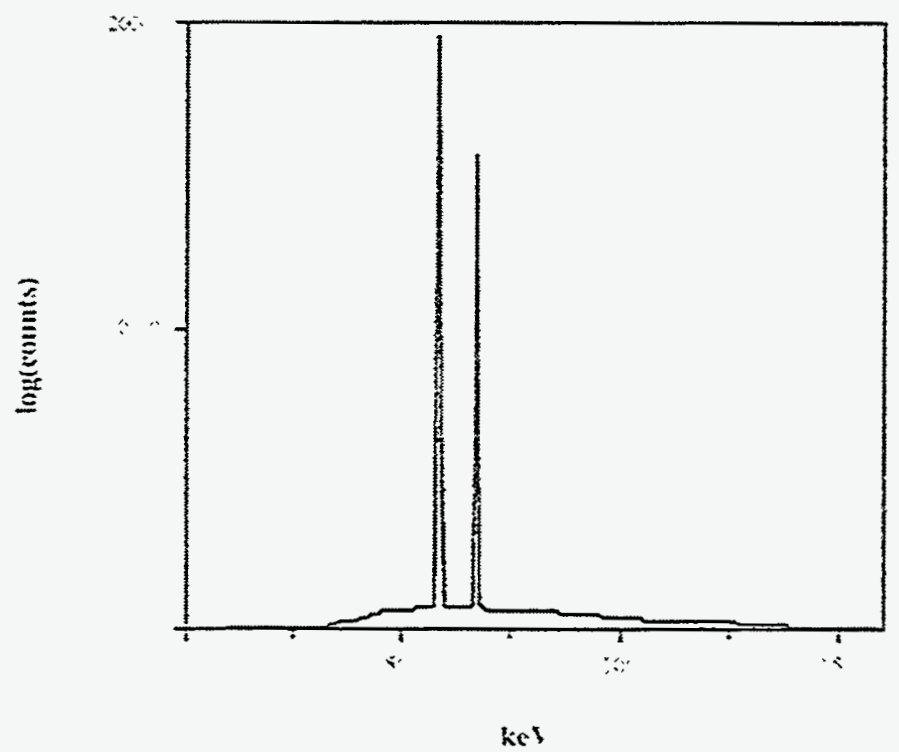

Figure 18. Calculated spectrum 2 from the hitered outpui of a tungsten anode $x-$ ray tube used to nroduce the radiogranhs shown in Figures 16 and 17 


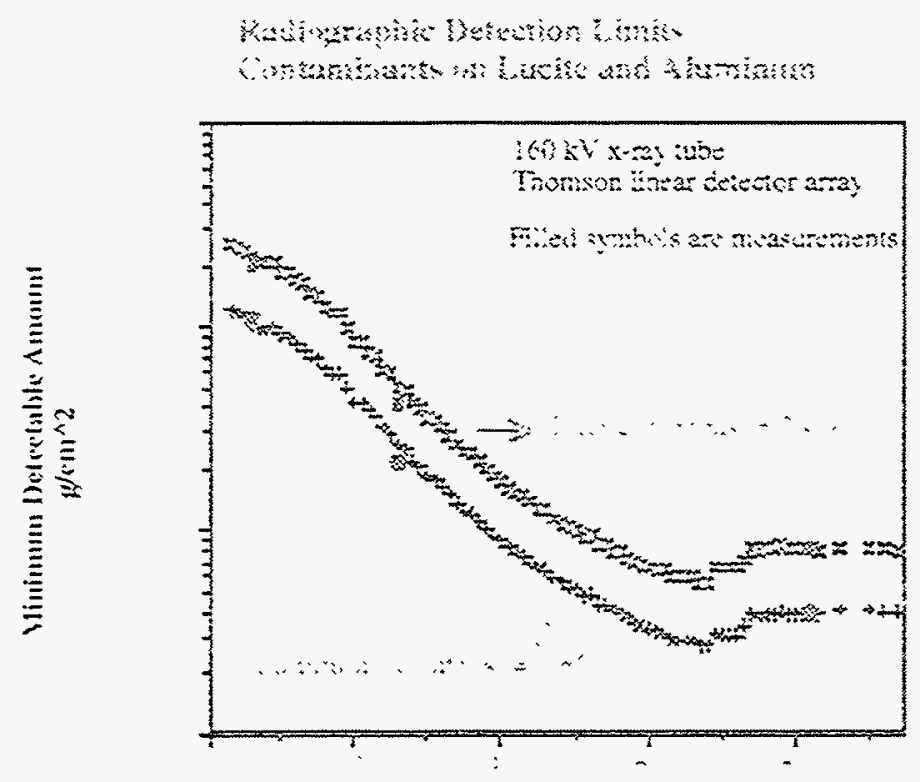

Atomic Number

Figure 19. Caiculated and measured radiogranhic detection limits for elements upon or within 2.5 $\mathrm{cm}$ aluminum of $15 \mathrm{~cm}$ Lucice. when iradiaied with the spectrum of $\mathrm{x}$-rays shown in Figure 18 . The measured vailes were taken from the radiographs shown in Figure 16 an Figure $i^{7}$.

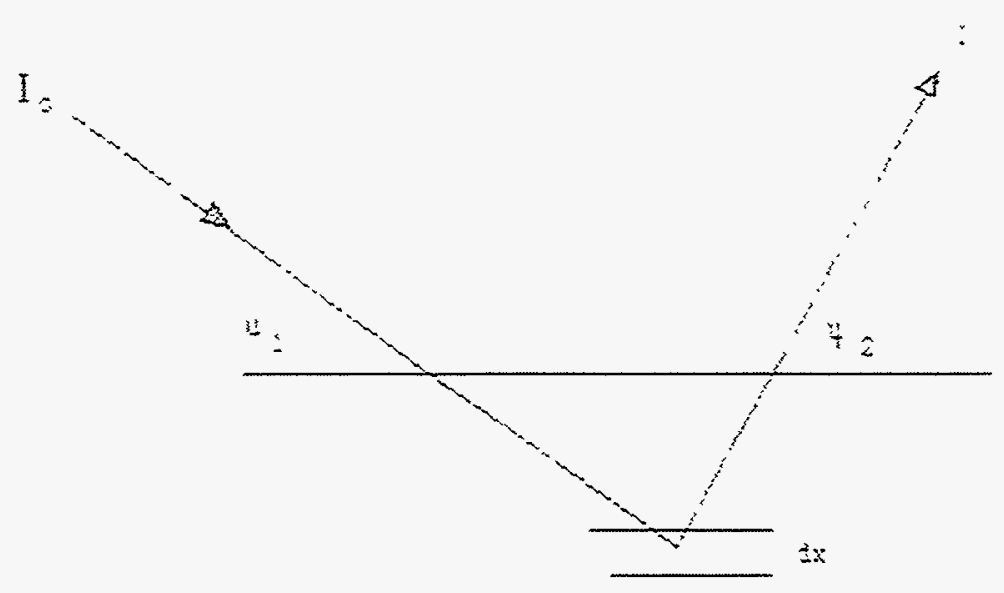

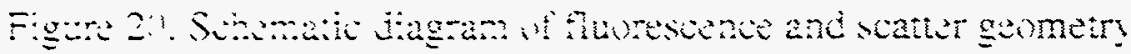




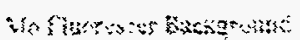

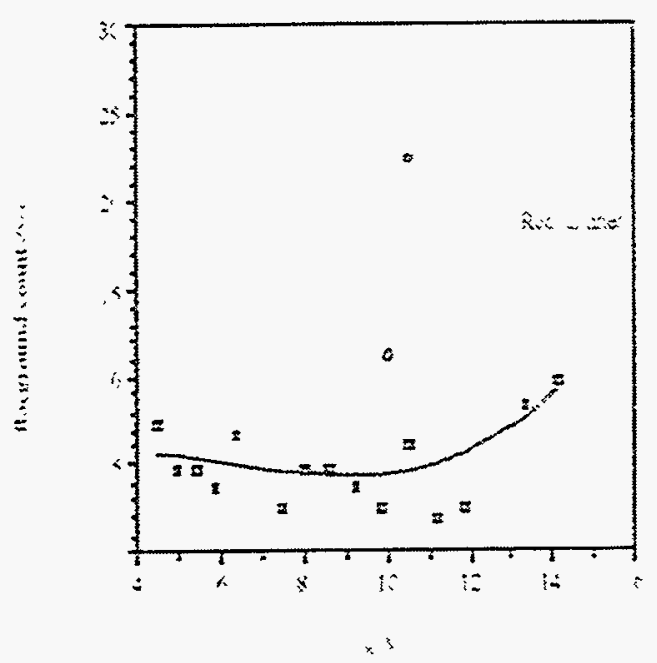

S3x:

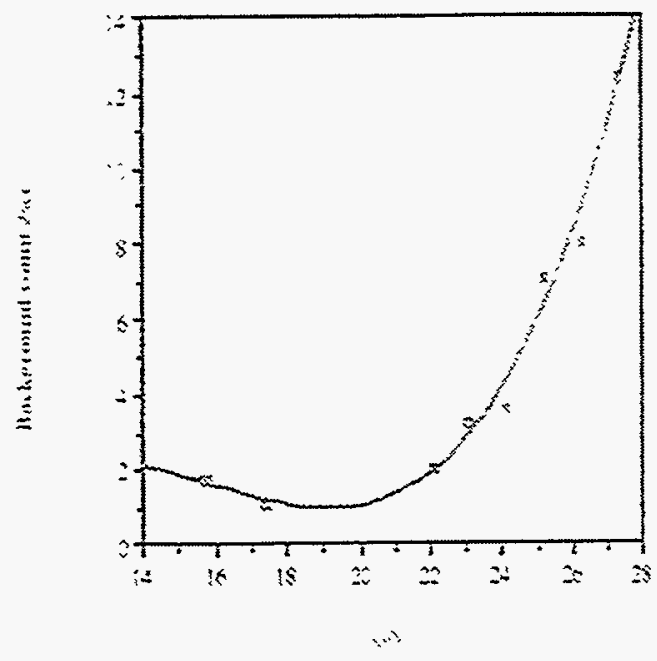

Figure 21. Estimated background from thin fims ${ }^{3}$. These functions were used to estimate the backgrounds for thick scatterers of plastic. aluminum. and steel.

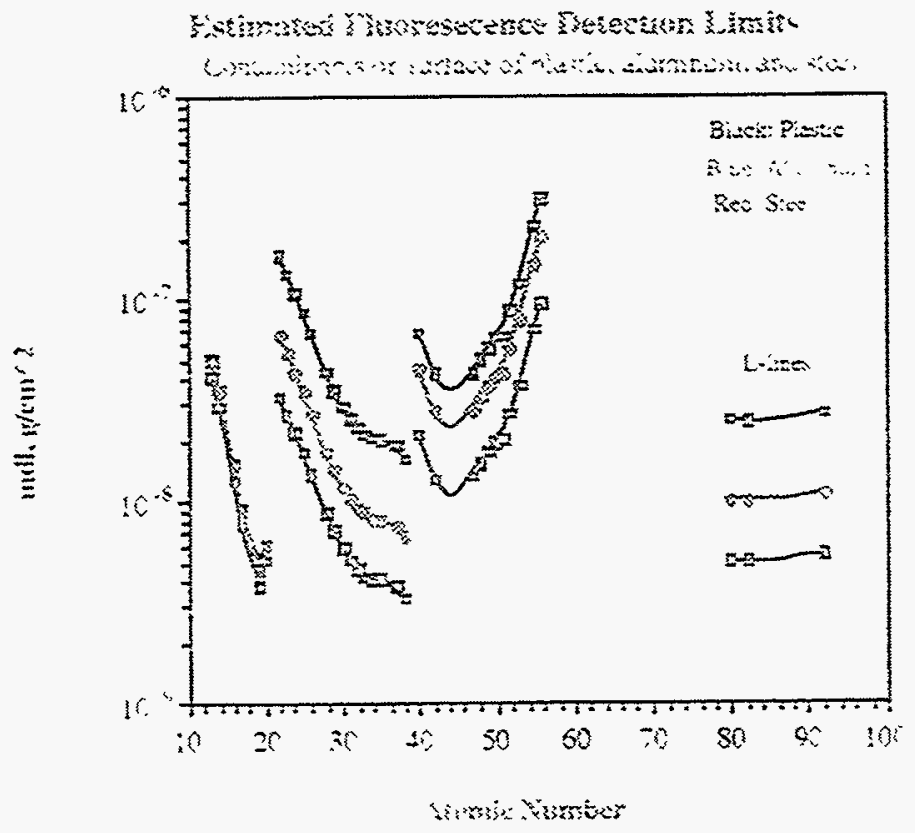

Figure 22. Estimated fluorescence minimum detection limits for contaminants on the surface of thick plastic. aluminum. and steel. Values are for an optimized $x$-ray spectrometer. using the secondary fuorescers titanium. molybdenum. and samarium. Estimates were made from published detecion limits for very thin substrates. ${ }^{3}$ 


$$
\text { บำ: }
$$

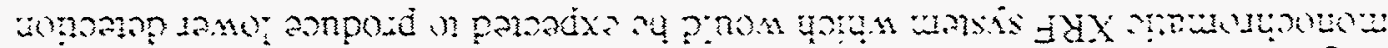

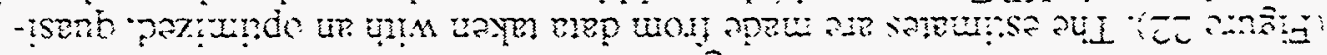

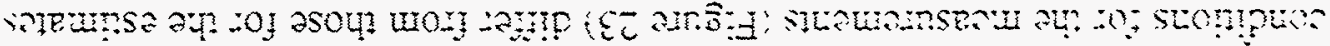

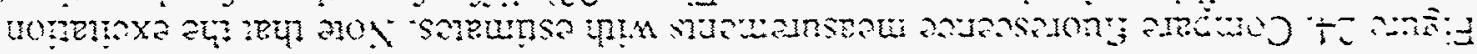

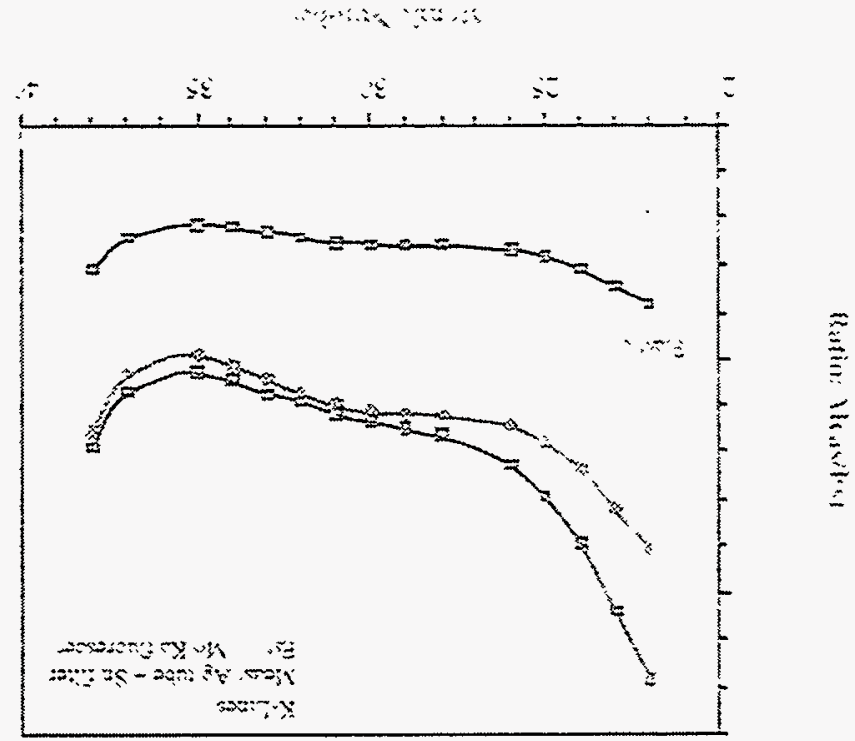

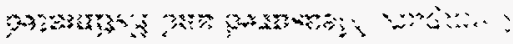

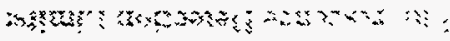

चall ur e wim

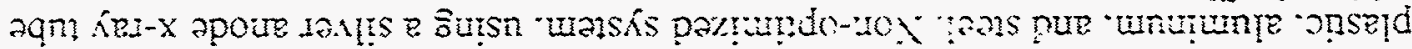

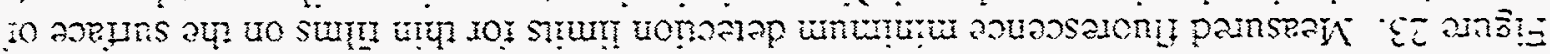

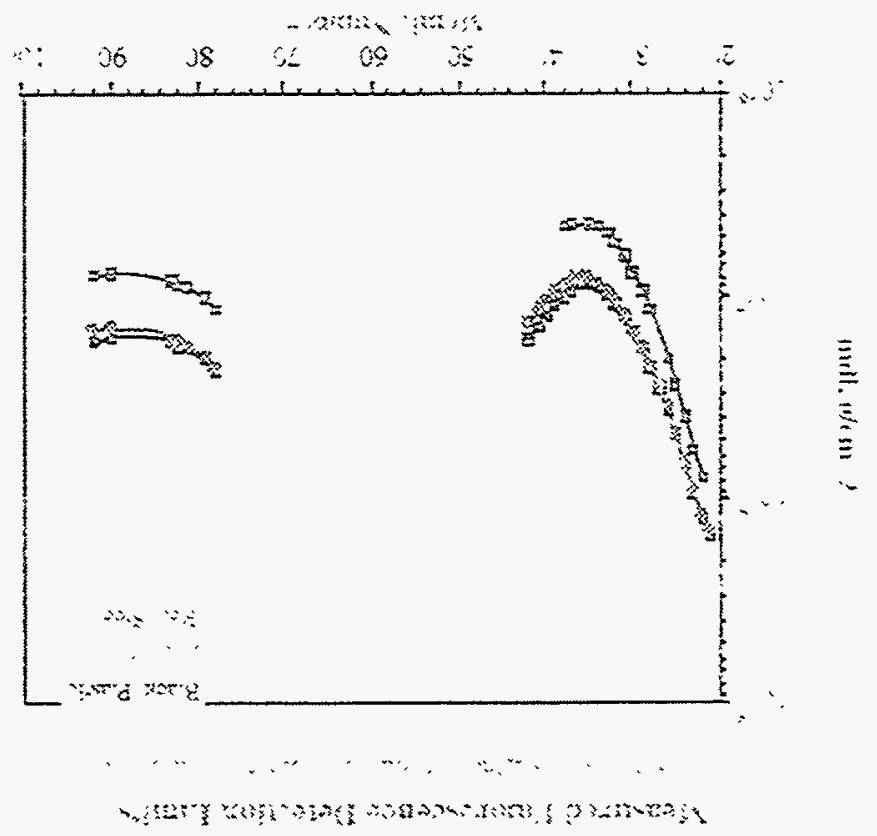



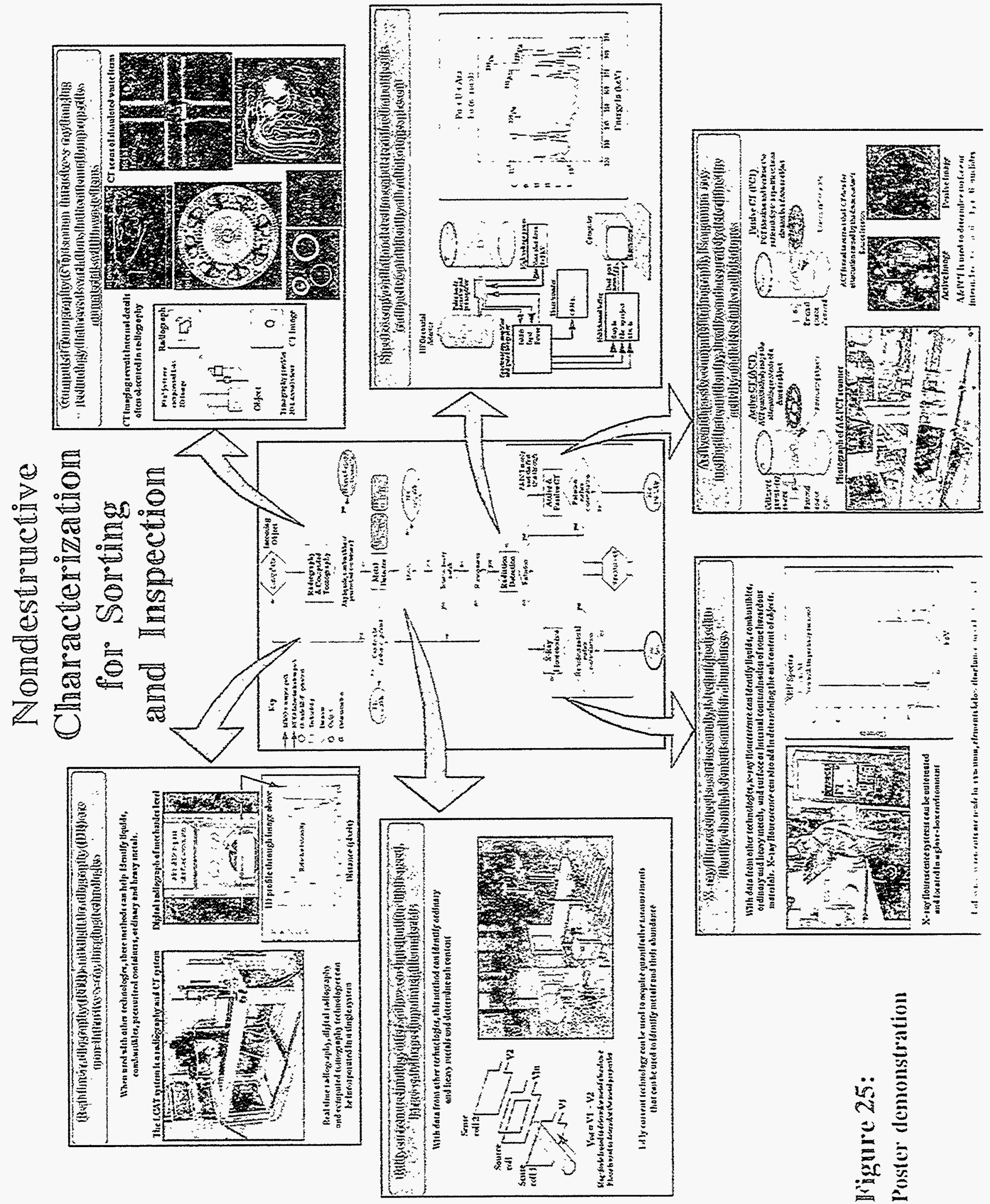

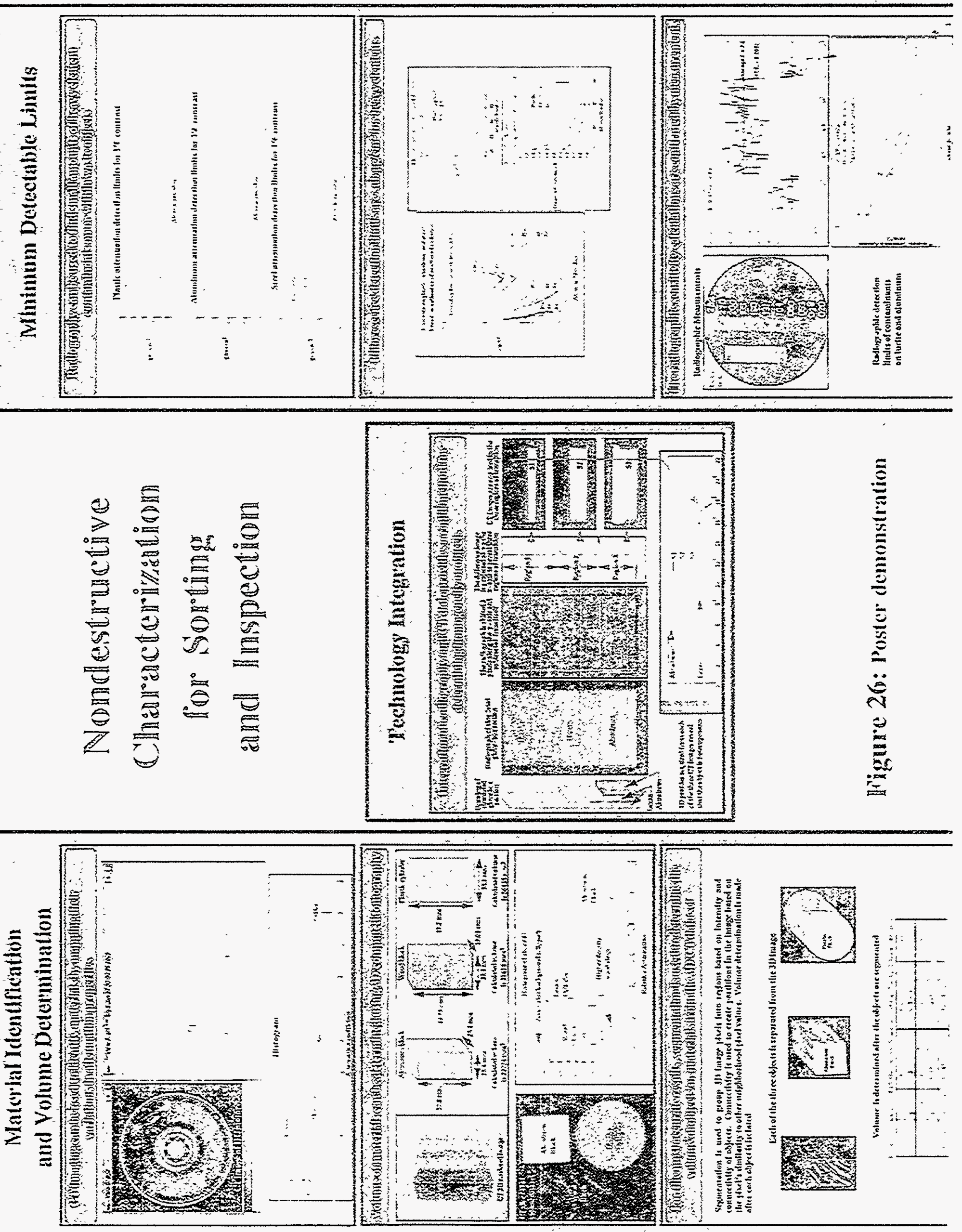


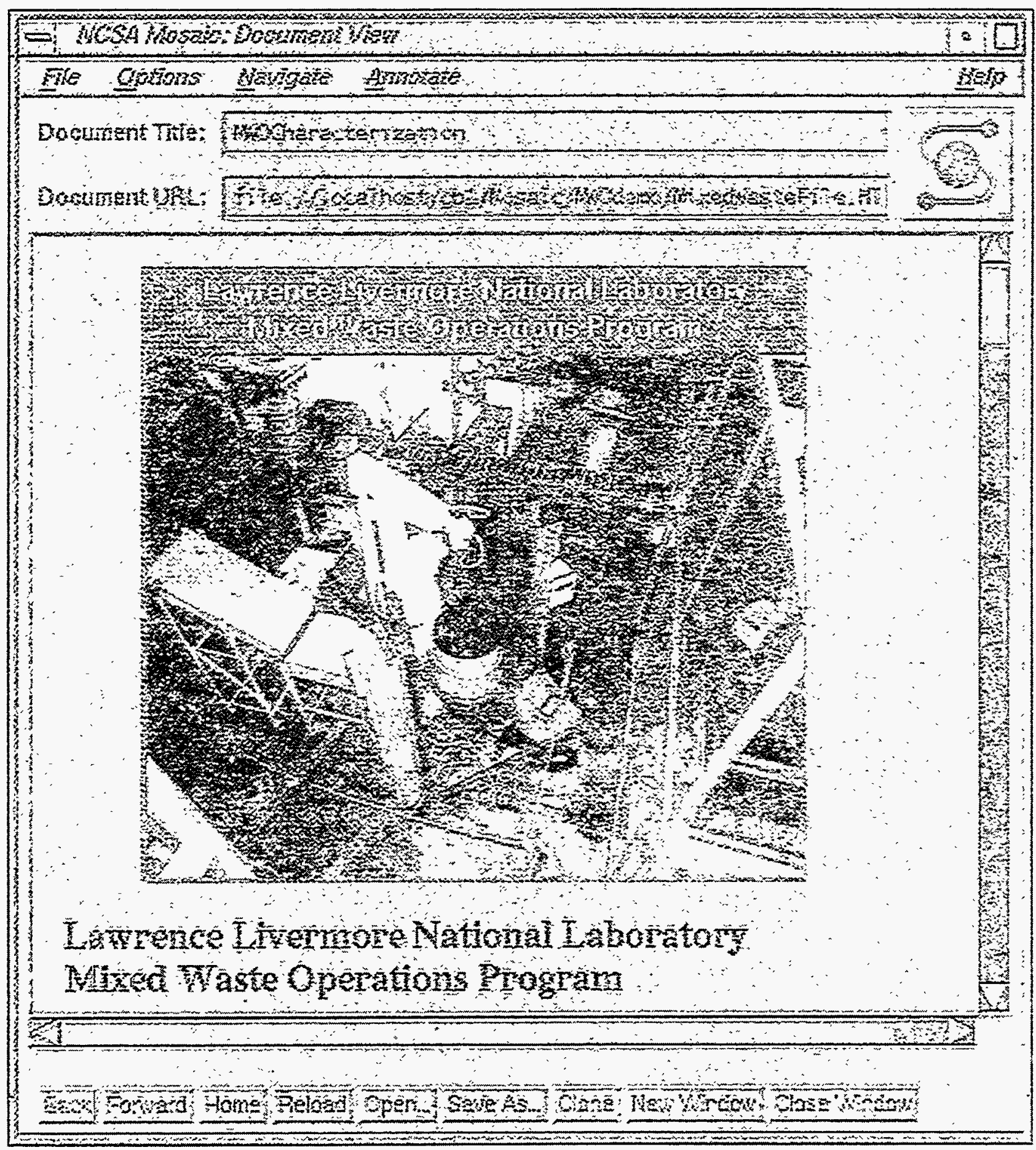

Figure 27: The Mosaic program was used to document a portion of the MII 0 project 